\title{
JIGSAW-GEO (1.0): locally orthogonal staggered unstructured grid generation for general circulation modelling on the sphere
}

\author{
Darren Engwirda ${ }^{1,2}$ \\ ${ }^{1}$ Department of Earth, Atmospheric and Planetary Sciences, Room 54-1517, Massachusetts Institute of Technology, \\ 77 Massachusetts Avenue, Cambridge, MA 02139-4307, USA \\ ${ }^{2}$ NASA Goddard Institute for Space Studies, 2880 Broadway, New York, NY 10025, USA \\ Correspondence to: Darren Engwirda (engwirda@mit.edu)
}

Received: 6 December 2016 - Discussion started: 22 December 2016

Revised: 24 April 2017 - Accepted: 25 April 2017 - Published: 6 June 2017

\begin{abstract}
An algorithm for the generation of non-uniform, locally orthogonal staggered unstructured spheroidal grids is described. This technique is designed to generate very high-quality staggered Voronoi-Delaunay meshes appropriate for general circulation modelling on the sphere, including applications to atmospheric simulation, ocean-modelling and numerical weather prediction. Using a recently developed Frontal-Delaunay refinement technique, a method for the construction of high-quality unstructured spheroidal Delaunay triangulations is introduced. A locally orthogonal polygonal grid, derived from the associated Voronoi diagram, is computed as the staggered dual. It is shown that use of the Frontal-Delaunay refinement technique allows for the generation of very high-quality unstructured triangulations, satisfying a priori bounds on element size and shape. Grid quality is further improved through the application of hill-climbing-type optimisation techniques. Overall, the algorithm is shown to produce grids with very high element quality and smooth grading characteristics, while imposing relatively low computational expense. A selection of uniform and non-uniform spheroidal grids appropriate for highresolution, multi-scale general circulation modelling are presented. These grids are shown to satisfy the geometric constraints associated with contemporary unstructured C-gridtype finite-volume models, including the Model for Prediction Across Scales (MPAS-O). The use of user-defined meshspacing functions to generate smoothly graded, non-uniform grids for multi-resolution-type studies is discussed in detail.
\end{abstract}

\section{Introduction}

The development of atmospheric and oceanic general circulation models based on unstructured numerical discretisation schemes is an emerging area of research. This trend necessitates the development of unstructured grid-generation algorithms designed to produce very high-resolution, guaranteedquality unstructured triangular and polygonal meshes that satisfy non-uniform mesh-spacing distributions and embedded geometrical constraints. This study investigates the applicability of a recently developed surface meshing algorithm (Engwirda and Ivers, 2016; Engwirda, 2016) based on restricted Frontal-Delaunay refinement and hill-climbing-type optimisation for this task.

\subsection{Semi-structured grids}

While simple structured grid types for the sphere can be obtained by assembling a uniform discretisation in spherical coordinates, the resulting latitude-longitude (lat-long) grid is often inappropriate for numerical simulation, due to the presence of strong grid singularities at the two poles. Such features manifest as local distortions in grid quality, consisting of regions of highly distorted quadrilateral grid cells. These low-quality elements can lead to a number of undesirable numerical effects - imposing restrictions on model time step and stability and compromising local spatial accuracy. As a result, a majority of currentgeneration general circulation models are instead based on semi-structured quadrilateral discretisation schemes, including the cubed-sphere (Adcroft et al., 2004; Marshall et al., 

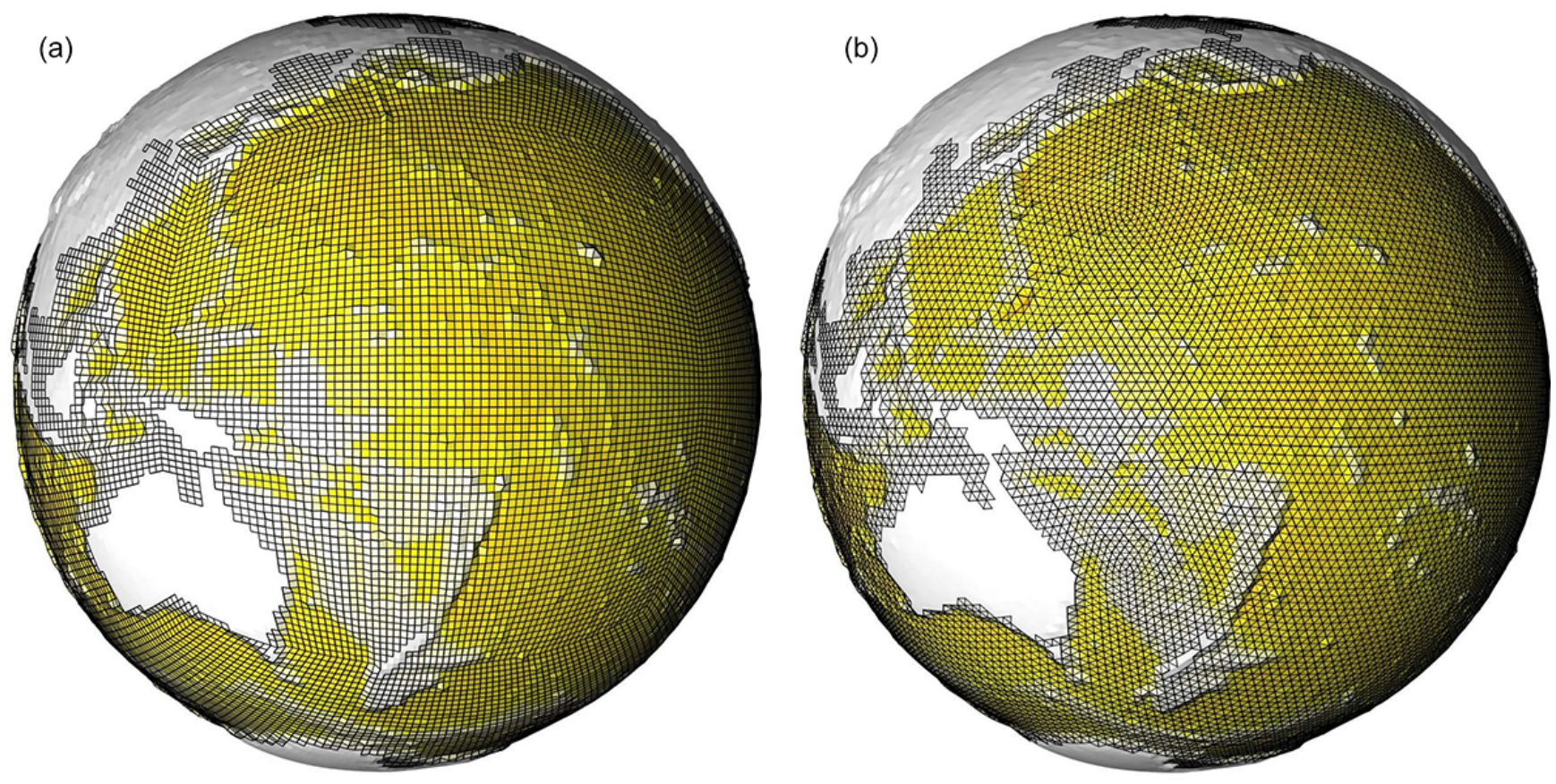

Figure 1. Conventional semi-structured meshes for the sphere, showing a cubed-sphere grid (a) and an icosahedral grid (b). Both grids were generated using equivalent target mean edge lengths and are coloured according to mean topographic height at grid cell centres. Topography is drawn using an exaggerated scale, with elevation from the reference geoid amplified by a factor of 20 in both cases.

1997; Putman and Lin, 2007) and tri-polar configurations (Murray, 1996; Madec et al., 2011; Bleck, 2002).

In the cubed-sphere framework, the spherical surface is decomposed into a cube-like topology, with each of the six quadrilateral faces discretised as a structured curvilinear grid. In such an arrangement, the two strong grid singularities of the lat-long configuration are replaced by eight weak discontinuities at the cube corners, leading to significant improvements in numerical performance. Putman and Lin (2007) presented detailed discussions of techniques for the generation and optimisation of cube-sphere-type grids. A regular gnomonic-type cubed-sphere grid is illustrated in Fig. 1a.

In the tri-polar grid, the present-day continental configuration is exploited to bury the singularities associated with a three-way polar decomposition of the sphere outside of the ocean mask. The resulting numerically active subset of the grid is well conditioned as a result. While such configurations are a popular choice for models designed for presentday Earth-centric ocean studies, the generality of these methods is clearly limited. In this study, we instead pursue the development of more general-purpose techniques, applicable to both oceanic and atmospheric modelling for general planetary and palaeo-Earth environments.

In addition to the standard cubed-sphere and tri-polar configurations, a second class of semi-structured spherical grid can be constructed through icosahedral-type decompositions (Heikes and Randall, 1995; Randall et al., 2002). In such cases, the primary grid is defined as a regular spherical trian- gulation, obtained through recursive bisection of the icosahedron. The associated staggered polygonal dual grid, consisting of hexagonal and pentagonal cells, is often used as a basis for finite-volume-type numerical schemes. This geometric duality is an example of the locally orthogonal VoronoiDelaunay-type grid staggering that forms the basis of this paper. Icosahedral-type grids provide a near-perfect tessellation of the sphere - free of topological discontinuities and/or geometrical irregularity. Such methods are applicable to both atmospheric- and oceanic-type simulations. A regular icosahedral-class grid is illustrated in Fig. $1 \mathrm{~b}$.

\subsection{Unstructured grids}

While the semi-structured grids described previously each provide effective frameworks for uniform resolution global simulation, the development of multi-resolution modelling environments requires alternative techniques. A range of new general circulation models, including the Finite Element Sea Ice-Ocean Model (FESOM) (Wang et al., 2014), the Finite Volume Community Ocean Model (FVCOM) (Chen et al., 2003, 2007; Lai et al., 2010), the Stanford Unstructured Non-hydrostatic Terrain-following Adaptive NavierStokes Simulator (SUNTANS) (Fringer et al., 2006; Vitousek and Fringer, 2014), and the Second-generation Louvainla-Neuve Ice-ocean Model (SLIM) (Bernard et al., 2007; Comblen et al., 2009), are based on semi-structured triangular grids, with the horizontal directions discretised according to an unstructured spherical triangulation, and the ver- 
tical direction represented as a stack of locally structured layers. The Model for Predication Across Scales (MPAS) (Skamarock et al., 2012; Ringler et al., 2013, 2008) adopts a similar arrangement, except that a locally orthogonal unstructured discretisation is adopted, consisting of both a Spherical Voronoi Tessellation (SVT) and its dual Delaunay triangulation. The use of fully unstructured representations, based on general tetrahedral and/or polyhedral grids, are also under investigation in the Fluidity framework (Ford et al., 2004a, b; Pain et al., 2005; Piggott et al., 2008). Such models all impose different requirements on the quality of the underlying unstructured grids, with some models, including FESOM, SLIM, and Fluidity, offering additionally flexibility. In all cases though, the performance of the numerical simulation can be expected to improve with increased grid quality - encouraging the search for optimised grid-generation algorithms. Further discussion of grid-quality constraints for general circulation modelling is presented in Sect. 2.

Existing approaches for unstructured grid generation on the sphere have focused on a number of techniques, including (i) the use of iterative, optimisation-type algorithms designed to construct Spherical Centroidal Voronoi Tessellations (SCVTs) (Jacobsen et al., 2013), and (ii) the adaptation of anisotropic two-dimensional meshing techniques (Lambrechts et al., 2008) that build grids in associated parametric spaces.

The MPI-SCVT algorithm (Jacobsen et al., 2013) is a massively parallel implementation of iterative Lloyd-type smoothing (Du et al., 1999) for the construction of SCVTs for use in the MPAS framework. In this approach, a set of vertices are distributed over the spherical surface and iteratively smoothed until a high-quality Voronoi tessellation is obtained. Specifically, each iteration repositions vertices to the centroids of their associated Voronoi cells and updates the topology of the underlying spherical Delaunay triangulation. While such an approach typically leads to the generation of high-quality centroidal Voronoi tessellations, the algorithm does not provide theoretical guarantees on minimum element quality, and often requires significant computational effort to achieve convergence. Additionally, current implementations of the MPI-SCVT algorithm do not provide a mechanism to constrain the grid to embedded features, such as coastal boundaries.

Lambrechts et al. (2008) presented an unstructured spherical triangulation framework using the general-purpose gridgeneration package Gmsh (Geuzaine and Remacle, 2009). In this work, unstructured spherical triangulations are generated for the world ocean using a parametric meshing approach. Specifically, a triangulation of the spherical surface is generated by mapping the full domain (including coastlines) on to an associated two-dimensional parametric space via stereographic projection. Importantly, as a result of the projection, the grids constructed in parametric space must be highly anisotropic, such that a well-shaped, isotropic triangulation is induced on the sphere. A range of existing two-dimensional anisotropic meshing algorithms are investigated, including Delaunay-refinement, advancing-front, and adaptation-type approaches. In particular, the algorithm is designed to ensure a faithful representation of complex coastal boundary conditions. While a detailed model of such constraints is often neglected in global simulations, resolution of these features is a key factor for regional and coastal models. Several additional algorithms support the generation of unstructured grids for such two-dimensional domains, including the ADmesh package (Conroy et al., 2012) and the Stomel library (Holleman et al., 2013).

The current study describes a new algorithm for the generation of guaranteed-quality spheroidal Delaunay triangulations and Voronoi tessellations - appropriate for a range of unstructured general circulation models. In this work, meshes are generated on the spheroidal surface directly, without need for local parameterisation or projection. Such an approach will be shown to exhibit significant flexibility immune to issues of coordinate singularity and/or continental configuration. The applicability of this approach to grid generation for imperfect spheres, including oblate spheroids and general ellipsoids is also discussed. Significant effort is invested to develop techniques designed to produce very highquality multi-resolution grids appropriate for contemporary unstructured C-grid models, including the MPAS.

The paper is organised as follows: an overview of grid generation for general circulation modelling is presented in Sect. 2, outlining various constraints and requirements on minimum grid quality. A description of the Frontal-Delaunay refinement and hill-climbing-type optimisation algorithms is given in Sects. 3 and 4. A set of uniform and non-uniform grids appropriate for high-resolution, multi-scale general circulation modelling are presented Sect. 5, alongside an analysis of computational performance and optimality. Avenues for future work are outlined in Sect. 6 .

\section{Grid generation for general circulation modelling}

Numerical formulations for atmospheric and/or oceanic general circulation modelling are typically based on a staggered grid configuration, with quantities such as fluid pressure, geopotential height, and density discretised using a primary control volume, and the fluid velocity field and vorticity distribution represented at secondary, spatially distinct grid points. Various structured staggered grid types are described by the well-known Arakawa schemes (Arakawa and Lamb, 1977).

The development of models based on unstructured grid types is an emerging area of research, and, as a result, a variety of numerical formulations are currently under investigation. In this study, the use of locally orthogonal grids appropriate for staggered unstructured C-grid schemes is pursued, as these methods represent a logical extension of conventional structured Arakawa-type techniques to the unstruc- 

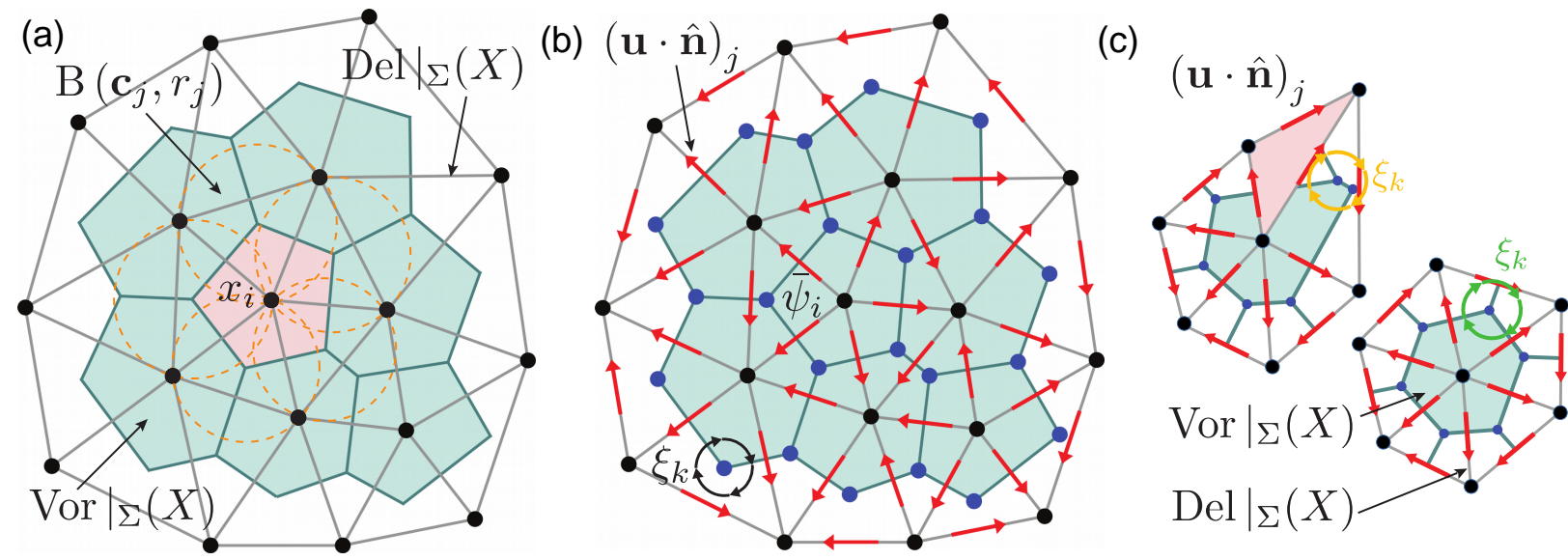

Figure 2. An anatomy of staggered unstructured grids for general circulation modelling, illustrating (a) locally orthogonal Voronoi-Delaunay grid staggering, (b) an unstructured C-grid-type numerical formulation, and (c) a comparison of well-centred and poorly staggered configurations. In panel (a), Voronoi polygons are formed by joining the centres of circumscribing balls $B\left(\boldsymbol{c}_{i}, r_{i}\right)$ associated with adjacent Delaunay triangles. In panel (b), the C-grid scheme consists of conservative cell-centred tracer quantities $\bar{\psi}_{i}$, edge-centred normal velocity components $(\boldsymbol{u} \cdot \hat{\boldsymbol{n}})_{j}$, and auxiliary vertex-centred vorticity variables $\xi_{k}$. In panel (c), the well-centred (lower) and poorly staggered (upper) configurations differ in the associativity of Voronoi vertices. In the well-centred configuration, Voronoi vertices are interior to their parent triangles. Reconstruction of the vertex-centred vorticity distribution can thus be achieved by integrating over the Delaunay triangles. In the poorly staggered configuration, a single Voronoi vertex is exterior to its parent triangle (shaded), leading to a breakdown in the reconstruction. Note that adjacent Voronoi-Delaunay edges do not intersect in the poorly staggered configuration.

tured setting. Such formulations require that the grids satisfy a local orthogonality constraint, with adjacent grid cell edges in the primary and secondary control volumes required to be mutually perpendicular. In the unstructured setting, it is known that the Delaunay triangulation and the Voronoi tessellation constitute a locally orthogonal staggered dual, leading to a natural framework for the construction of such meshes.

Given a Delaunay triangulation $\left.\operatorname{Del}\right|_{\Sigma}(X)$ of the surface $\Sigma$, the associated Voronoi diagram $\left.\operatorname{Vor}\right|_{\Sigma}(X)$ can be constructed by exploiting this underlying geometric duality. Each edge in the Delaunay triangulation is associated with a dual edge in the Voronoi diagram, spanning between the centres of the circumscribing balls $B\left(\boldsymbol{c}_{j}, r_{j}\right)$ associated with the two adjacent Delaunay triangles. The full Voronoi diagram is assembled from these edges - consisting of a set of (convex) polygonal grid cells centred on each vertex in the underlying surface triangulation. See Fig. 2a for an example of this unstructured Voronoi-Delaunay-type grid staggering.

In Fig. 2b, an unstructured variant of the widely used Arakawa C-grid scheme is described, with fluid pressure, geopotential height, and density degrees of freedom placed within the primary Voronoi control volumes, and a set of orthogonal velocity vectors positioned along Delaunay edges. Additional vorticity degrees of freedom are located at the vertices of the Voronoi grid cells. Such an arrangement facilitates the construction of a standard conservative finite-volume-type scheme for the transport of cell-centred fluid properties, and a mimeticclass

(Lipnikov et al., 2014; Bochev and Hyman, 2006) finite-difference formulation for the evolution of velocity components. Overall, this scheme is known to posses a variety of desirable conservation properties, conserving mass, potential vorticity and enstrophy, and preserving geostrophic balance (Ringler et al., 2010). This unstructured $\mathrm{C}$-grid scheme is currently employed in the MPAS for both atmospheric and oceanic modelling (Skamarock et al., 2012; Ringler et al., 2013, 2008).

While numerically elegant, such schemes are not applicable to general unstructured Voronoi-Delaunay grids - requiring that a number of auxiliary geometrical constraints also be satisfied. Specifically, such schemes necessitate the use of grids that are not only locally orthogonal but also well centred and mutually centroidal. These additional conditions are constraints on the geometry of the underlying Delaunay triangles and Voronoi polygons.

A well-centred Delaunay grid is one in which all dual Voronoi vertices lie within the interior of their associated Delaunay triangles. Such a condition guarantees that adjacent Delaunay and Voronoi edges intersect - inducing a dual structure that is nicely staggered. In the context of the unstructured C-grid scheme described previously, this configuration guarantees that a consistent stencil exists for reconstruction of the discrete vorticity variable. In practice, the generation of well-centred grids is known to be a particularly onerous task (VanderZee et al., 2008; Vanderzee et al., 2010), requiring that the triangulation consist of all acute elements. Degradation of the vorticity reconstruction for poorly staggered grids is described in Fig. 2c. 
Delaunay grids are centroidal when their primary and secondary vertices lie at the centres of mass of their associated dual grid cells, with the vertices of the Voronoi polygons lying at the centroids of the Delaunay triangles and visa versa. Such a condition is effectively an implicit constraint on the regularity of the grid, with strongly centroidal meshes typically associated with improved grid cell shapes. In the limit, a perfectly centroidal and well-centred tessellation can be assembled from equilateral triangles and regular hexagons. Centroidal grids typically lead to very high-quality numerical discretisations, with grids containing near-perfect element configurations achieving optimal convergence rates. The unstructured C-grid scheme described previously is known to achieve second-order accurate convergence when applied to centroidal Voronoi grids (Ringler et al., 2010).

The generation of locally orthogonal, well-centred, and centroidal unstructured grids is a difficult task, imposing a heavy burden on the underlying mesh generation algorithm. While a number of techniques for unstructured grid generation currently exist, as per Sect. 1, the author is not aware of any that are successful in generating the very high-quality grids required by unstructured C-grid-type general circulation models. As a result, throughout the remainder of this paper, the development of new methods for the generation of such tessellations is pursued in detail.

\section{A restricted Frontal-Delaunay refinement algorithm}

The task is to generate very high-resolution, guaranteedquality unstructured Delaunay triangulations for planetary atmospheres and/or oceans. These grids will form a baseline for the hill-climbing mesh-optimisation techniques presented in subsequent sections. Such grids are required to satisfy a number of constraints, including bounds on minimum element quality and adherence to user-defined mesh-spacing distributions. In this work, the applicability of a recently developed Frontal-Delaunay surface meshing algorithm (Engwirda and Ivers, 2016; Engwirda, 2016) is investigated for this task.

An unstructured Delaunay triangulation of the reference spheroid associated with a general planetary geometry is sought. In a general form, this reference surface can be expressed as an axis-aligned triaxial ellipsoid

$\sum_{i=1}^{3}\left(\frac{x_{i}}{r_{i}}\right)^{2}=1$,

where the $x_{i}$ 's are the Cartesian coordinates in a locally aligned coordinate system, and the scalars $r_{i}>0$ are its principal radii. Such a definition can be used to represent ellipsoidal surfaces in general position, based on the application of additional rigid-body translations and rotations. Note that while grid generation for global climate modelling is often restricted to spherical surfaces (e.g. $r_{1,2,3}=$
$6371 \mathrm{~km})$ this formulation facilitates mesh generation for general spheroidal and ellipsoidal domains.

\subsection{Preliminaries}

Before describing the Frontal-Delaunay refinement algorithm in full, several key properties of the underlying Delaunay triangulation and Voronoi tessellation are briefly reviewed. A full account of these structures is not presented here; instead, the reader is referred to the detailed theoretical exposition presented in, for example, Cheng et al. (2013).

The Delaunay triangulation $\operatorname{Del}(X)$ associated with a set of points $X \in \mathbb{R}^{d}$ is characterised by the empty-circle criterion - requiring that the set of circumscribing spheres $B\left(\boldsymbol{c}_{i}, r_{i}\right)$ associated with each Delaunay triangle $\tau_{i} \in \operatorname{Del}(X)$ be empty of all points other than its own vertices. It is well known that for tessellations restricted to two-dimensional manifolds, the Delaunay triangulation leads to a maximisation of the minimum enclosed angle in the grid (Cheng et al., 2013). Such behaviour is clearly beneficial when seeking to construct high-quality triangular meshes.

The Voronoi tessellation $\operatorname{Vor}(X)$ is the geometric dual associated with the Delaunay triangulation, consisting of a set of convex polygonal cells formed by connecting the centres of adjacent circumscribing balls - the element circumcentres $\boldsymbol{c}_{i}$ 's. The Voronoi tessellation represents a closest-point map for the points in $X$, with each Voronoi cell $v_{c} \in \operatorname{Vor}(X)$ defining the convex region adjacent to a given vertex $\boldsymbol{x}_{i} \in X$ for which $\boldsymbol{x}_{i}$ is the nearest point. Importantly, the VoronoiDelaunay grid staggering, defines a locally orthogonal arrangement, in which grid cell edges in the Voronoi tessellation are orthogonal to adjacent edges in the underlying Delaunay triangulation.

\subsection{Restricted Delaunay triangulation}

In this study, grid generation is carried out directly on the spheroidal geometry by making use of restricted Delaunay mesh generation techniques. Specifically, given a reference surface $\Sigma$, grid generation proceeds to discretise the surface into a mesh of triangles. In the restricted Delaunay framework, a full-dimensional Delaunay tessellation $\operatorname{Del}(X)$ (i.e. a tetrahedral tessellation) is maintained, with the surface triangulation represented as a subset of the tetrahedral faces. The restricted Delaunay surface triangulation $\left.\operatorname{Del}\right|_{\Sigma}(X)$ is said to be embedded in $\operatorname{Del}(X)$ as a result. Use of this fully three-dimensional approach elides any reliance on local parametric projections.

Definition 1 (restricted Delaunay tessellation). Let $\Sigma$ be a smooth surface embedded in $\mathbb{R}^{3}$. Let $\operatorname{Del}(X)$ be a full-dimensional Delaunay tetrahedralisation of a point-wise sample $X \subseteq \Sigma$ and $\operatorname{Vor}(X)$ be the associated Voronoi tessellation. The restricted Delaunay surface triangulation $\left.\operatorname{Del}\right|_{\Sigma}(X)$ is a sub-complex of $\operatorname{Del}(X)$ including any triangle 
$f_{i} \in \operatorname{Del}(X)$ associated with an intersecting Voronoi edge $v_{e} \in \operatorname{Vor}(X)$ such that $v_{e} \cap \Sigma \neq \varnothing$.

The development of restricted Delaunay techniques for general mesh generation applications has been the subject of previous research, and a detailed discussion of such concepts is presented elsewhere. The reader is referred to the original work of Edelsbrunner and Shah (1997) or the detailed reviews presented in Cheng et al. (2013) for additional details and mathematical background.

In the context of this work, it is sufficient to note that the restricted Delaunay framework provides a convenient mechanism for the construction of grids on the spheroidal surface $\Sigma$, without requiring the introduction of parametric mappings or anisotropic metrics. Implementation of the restricted Delaunay scheme requires the definition of a single geometric predicate - a local comparison used to determine which faces of the underlying tetrahedral complex lie on the spheroidal surface. Specifically, intersections between the edges of the full-dimensional Voronoi complex and the underlying surface $\Sigma$ are computed. Triangles associated with non-empty intersections $\left.\operatorname{Vor}\right|_{f}(X) \cap \neq \varnothing$ form part of the surface mesh. In this study, these comparisons are computed analytically, following standard spheroidal trigonometric manipulations, as detailed in Appendix A. See Fig. 3 for a detailed schematic.

\subsection{Mesh-spacing functions}

The local density of the mesh can be controlled via a userspecified mesh-spacing function $\bar{h}(\boldsymbol{x}): \mathbb{R}^{3} \rightarrow \mathbb{R}^{+}$, where $\bar{h}(\boldsymbol{x})$ defines the target edge lengths at all points on the surface $\Sigma$. In this work, mesh-spacing functions are specified as a discrete set of target values $\bar{h}_{i, j}$, defined on a simple background lat-long grid $\mathcal{G}$. The continuous mesh-spacing function $\bar{h}(\boldsymbol{x})$ is reconstructed using bilinear interpolation. As will be illustrated in subsequent sections, such an approach provides support for a wide range of mesh-spacing definitions, including those derived from high-resolution topographic data (Amante and Eakins, 2009) or solution-adaptive metrics.

In order to generate high-quality grids, it is necessary to ensure that the imposed mesh-spacing function is sufficiently smooth. Rather than requiring the user to accommodate such constraints, a Lipschitz smoothing process is adopted here. Following the work of Persson (2006), a gradient-limited mesh-spacing function $\bar{h}^{\prime}(\boldsymbol{x})$ is constructed by constraining the allowable spatial fluctuation over each element in the background grid $\mathcal{G}$. In this study, a scalar smoothing parameter $g \in \mathbb{R}^{+}$is used to limit variation, such that

$\bar{h}^{\prime}\left(\boldsymbol{x}_{i}\right) \leq \bar{h}^{\prime}\left(\boldsymbol{x}_{j}\right)+g \cdot \operatorname{dist}\left(\boldsymbol{x}_{i}, \boldsymbol{x}_{j}\right)$,

for all adjacent vertex pairs $\boldsymbol{x}_{i}, \boldsymbol{x}_{j}$ in the underlying grid $\mathcal{G}$. The gradient-limited mesh-spacing function $\bar{h}^{\prime}(\boldsymbol{x})$ becomes more uniform as $g \rightarrow 0$. In this work, gradient limiting is implemented following a fast-marching method, as described in Persson (2006).

\subsection{Grid-quality metrics}

The quality of a mesh can be evaluated using various grid-quality metrics. Here, a number of standard measures are introduced.

Definition 2 (radius-edge ratio). Given a surface triangle $\left.f_{i} \in \operatorname{Del}\right|_{\Sigma}(X)$, its radius-edge ratio, $\rho\left(f_{i}\right)$, is given by

$\rho\left(f_{i}\right)=\frac{r_{i}}{\left\|\boldsymbol{e}_{\min }\right\|}$,

where $r_{i}$ is the radius of the circumscribing ball associated with $f_{i}$ and $\left\|\boldsymbol{e}_{\min }\right\|$ is the length of its shortest edge.

The radius-edge ratio is a measure of element shape quality, achieving a minimum of $\frac{1}{\sqrt{3}}$ for equilateral triangles and increasing as elements tend toward degeneracy. It is directly related to the minimum-angle $\theta_{\min }$ subtended by triangle edges, such that $\theta_{\min }=\arcsin \left(\frac{1}{2 \bar{\rho}}\right)$. Due to the summation of angles in a triangle, this relationship extends to the maximum angle, with $\theta_{\max } \leq \pi-2 \theta_{\min }$.

Definition 3 (area-length ratio). Given a surface triangle $\left.f_{i} \in \operatorname{Del}\right|_{\Sigma}(X)$, its area-length ratio, $a\left(f_{i}\right)$, is given by

$a\left(f_{i}\right)=\frac{4 \sqrt{3}}{3} \frac{A_{f}}{\left\|\boldsymbol{e}_{\mathrm{rms}}\right\|^{2}}$,

where $A_{f}$ is the signed area of $f_{i}$ and $\left\|\boldsymbol{e}_{\mathrm{rms}}\right\|$ is the rootmean-square edge length.

The area-length ratio is a robust, scalar measure of element shape quality, and is typically normalised to achieve a score of +1 for ideal elements. The area-length ratio decreases with increasing distortion, achieving a score of +0 for degenerate elements and -1 for tangled elements with reversed orientation.

Definition 4 (relative-edge length). Given an edge in the surface tessellation $\left.e_{j} \in \operatorname{Del}\right|_{\Sigma}(X)$, its relative-edge length, $h_{\mathrm{r}}\left(e_{j}\right)$, is given by

$h_{\mathrm{r}}\left(e_{j}\right)=\frac{\left\|\boldsymbol{e}_{j}\right\|}{\bar{h}\left(\boldsymbol{x}_{\mathrm{m}}\right)}$,

where $\left\|\boldsymbol{e}_{j}\right\|$ is the length of the $j$-th edge and $\bar{h}\left(\boldsymbol{x}_{\mathrm{m}}\right)$ is the value of the mesh-spacing function sampled at the edge midpoint.

The relative length $h_{\mathrm{r}}\left(e_{j}\right)$ is a measure of mesh-spacing conformance, expressing the ratio of actual-to-desired edgelength. A value of $h_{\mathrm{r}}\left(e_{j}\right)=1$ indicates perfect mesh-spacing conformance. 


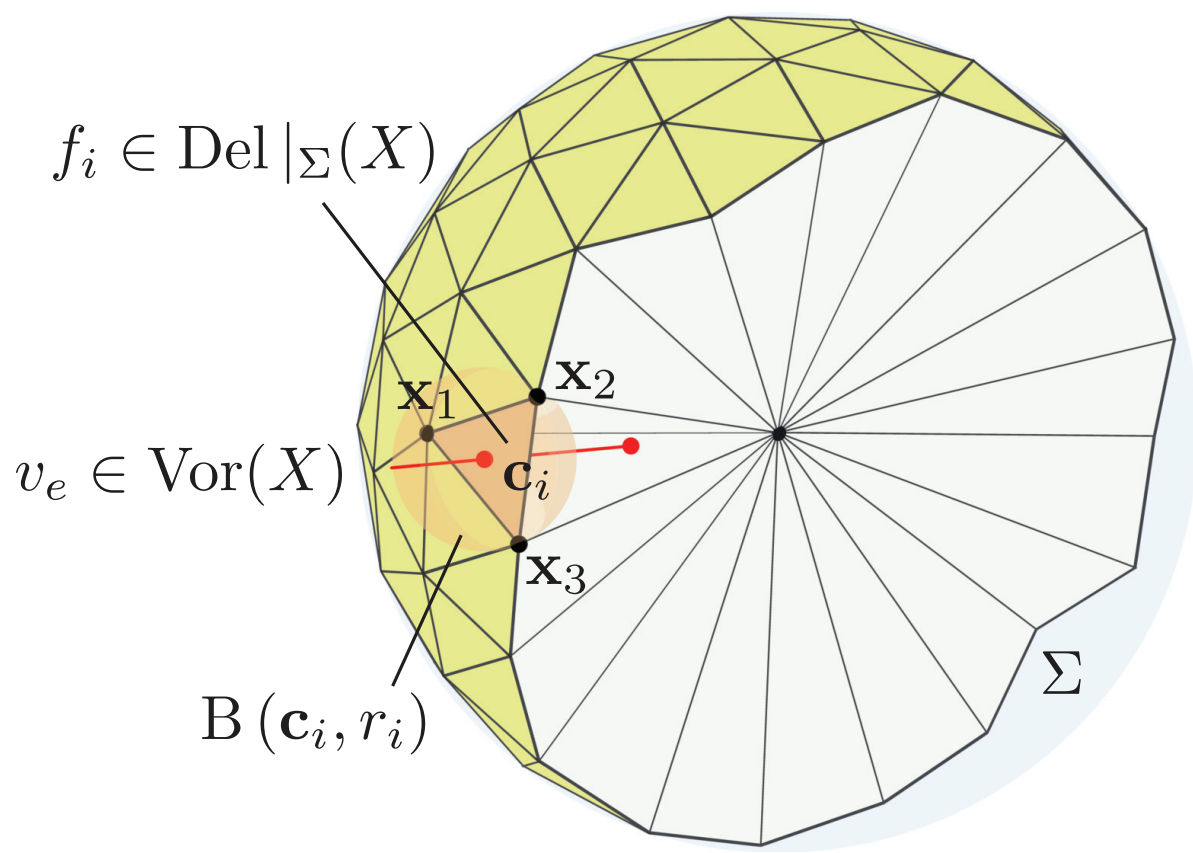

Figure 3. Illustration of the geometrical predicates used to define the restricted Delaunay surface triangles $\left.f_{i} \in \operatorname{Del}\right|_{\Sigma}(X)$, showing details of the intersecting Voronoi edge $v_{e} \in \operatorname{Vor}(X)$ associated with the surface triangle $\left.f_{i} \in \operatorname{Del}\right|_{\Sigma}(X)$ and its associated surface ball $B\left(c_{i}, r_{i}\right)$. Note that the full three-dimensional Delaunay tessellation $\operatorname{Del}(X)$ is a tetrahedral complex that fills the interior of the spheroid.

\subsection{Restricted Frontal-Delaunay refinement}

A high-quality triangular surface mesh is generated on the spheroidal reference surface $\Sigma$ using a Frontal-Delaunay variant of the conventional restricted Delaunay refinement algorithm (Boissonnat and Oudot, 2003, 2005; Jamin et al., 2015; Cheng et al., 2007, 2010). This technique is described by the author in detail in Engwirda and Ivers (2016); Engwirda (2016) and differs from standard Delaunay refinement approaches in terms of the strategies used for the placement of new vertices. Specifically, the Frontal-Delaunay algorithm employs a generalisation of various off-centre pointplacement techniques (Rebay, 1993; Erten and Üngör, 2009), designed to position vertices such that element-quality and mesh-size constraints are satisfied in a locally optimal fashion. Previous studies have shown that such an approach typically leads to substantial improvements in mean element quality and mesh smoothness.

While a variety of solutions for the surface meshing problem have been described previously (e.g. Frey and George, 2000; Rypl and Krysl, 1997; Schreiner et al., 2006; Löhner, 1996), the restricted Frontal-Delaunay algorithm presented here has been found to offer a unique combination of characteristics - combining the smooth, high-quality grid-generation capabilities of an advancing-front approach, with the theoretical robustness and provable guarantees associated with conventional Delaunay refinement techniques. Specifically, the Frontal-Delaunay algorithm described here is known to generate grids with very high mean element qual- ity, bounded minimum and maximum angles, tight conformance to grid-spacing constraints, and provable guarantees on topological consistency and convergence. A full description of the algorithm, including detailed discussions of its theoretical foundations and proofs of worst-case grid-quality bounds, can be found in Engwirda and Ivers (2016); Engwirda (2016).

Given a user-defined mesh-spacing function $\bar{h}(\boldsymbol{x})$ and an upper bound on the element radius-edge ratios $\bar{\rho} \geq$ 1, the Frontal-Delaunay algorithm proceeds to sample the spheroidal surface $\Sigma$ by refining any surface triangle that violates either the mesh-spacing or element-quality constraints. The full algorithm is described in Algorithm 1, and detailed snap shots of the refinement process are shown in Fig. 4.

Initially, the surface is seeded with a set of 12 vertices to form a standard icosahedron. Refinement then proceeds to insert a new vertex at the off-centre refinement point associated with each element to be eliminated. Refinement continues until all constraints are satisfied. The refinement process is priority scheduled, with triangles $\left.f_{i} \in \operatorname{Del}\right|_{\Sigma}(X)$ ordered according to their radius-edge ratios $\rho\left(f_{i}\right)$. This ordering ensures that the element with the worst ratio is refined at each iteration. Additionally, triangles are subject to a frontal filtering - requiring that a low-quality element be adjacent to a converged triangle before being considered for refinement. This logic helps the algorithm mimic the behaviour of advancing-front-type techniques, with new vertices and elements expanding from initial seeds. Upon termination, the resulting surface triangulation is guaranteed to contain nicely 


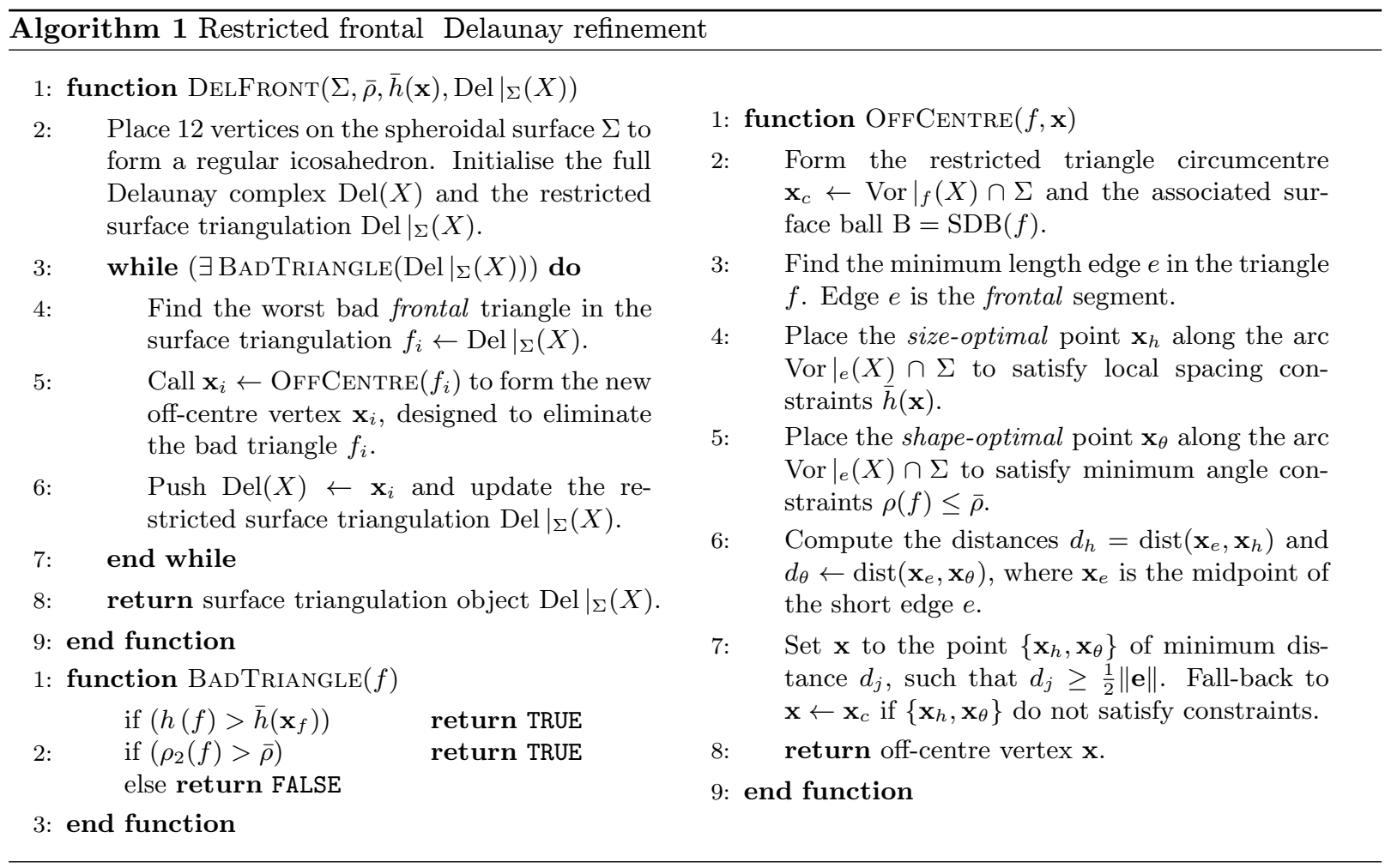

shaped elements, satisfying both the radius-edge constraints $\rho\left(f_{i}\right) \leq \bar{\rho}$ and mesh-spacing bounds $h\left(f_{i}\right) \leq \bar{h}\left(\boldsymbol{x}_{f}\right)$. Setting $\bar{\rho}=1$ guarantees that element angles are bounded above $30^{\circ}$, ensuring that the grid does not contain any highly distorted triangles.

\subsection{Off-centre point placement}

The performance of the Frontal-Delaunay algorithm hinges on the use of off-centre refinement rules - locally optimal point-placement strategies designed to create very highquality vertex distributions. A set of candidate off-centre points are considered at each new vertex insertion. Type I vertices, $\boldsymbol{x}_{c}$, are equivalent to conventional element circumcentres (positioned at the centre of the associated element circumballs), and are used to preserve global convergence. Type II vertices, $\boldsymbol{x}_{h}$, are size-optimal points, and are designed to satisfy grid-spacing constraints in a locally optimal fashion. Type III vertices, $\boldsymbol{x}_{\theta}$, are shape-optimal points, and are designed to ensure minimum-angle bounds are satisfied in a worst-first manner. The type II and type III strategies employed here can be seen as a generalisation of the two-dimensional off-centre techniques presented by Rebay (1993) and Erten and Üngör (2009), respectively. See Fig. 5 for additional detail.

Given a low-quality triangle $\left.f_{i} \in \operatorname{Del}\right|_{\Sigma}(X)$ to be eliminated, the type II and type III vertices $\boldsymbol{x}_{h}$ and $\boldsymbol{x}_{\theta}$ are positioned along the intersection of an adjacent segment of the
Voronoi complex and the spheroidal surface. This intersection $\left.\operatorname{Vor}\right|_{e}(X) \cap \Sigma$, defines a frontal curve inscribed on $\Sigma-$ a locally optimal geodesic segment on which to insert new vertices. Here, $\left.\operatorname{Vor}\right|_{e}(X)$ is the polygonal face of the Voronoi complex associated with the short edge $e_{0} \in f_{i}$. In the context of conventional advancing-front-type methods, the edge $e_{0}$ would denote the current frontal segment about which vertex insertion occurs.

The points $\boldsymbol{x}_{h}$ and $\boldsymbol{x}_{\theta}$ are positioned to form new candidate triangles about the frontal edge $e_{0}$, such that local constraints are satisfied optimally. The size-optimal point $\boldsymbol{x}_{h}$ is positioned to adhere to local grid-spacing constraints, ensuring that new edges satisfy $\left\|\boldsymbol{e}_{i}\right\| \leq \bar{h}\left(\boldsymbol{m}_{i}\right)$, where the $\boldsymbol{m}_{i}$ 's are the edge midpoints. These constraints can be solved for an associated altitude

$a_{h}=\min \left(\tilde{a}_{h}, \frac{1}{2} \sqrt{3} \tilde{h}\right)$,

where

$\widetilde{a}_{h}=\sqrt{\widetilde{h}^{2}-\frac{1}{2}\left\|\boldsymbol{e}_{0}\right\|^{2}}, \quad \widetilde{h}=\frac{\bar{h}\left(\boldsymbol{m}_{1}\right)+\bar{h}\left(\boldsymbol{m}_{2}\right)}{2}$.

Similarly, the shape-optimal point is positioned to adhere to minimum-angle constraints, sliding $\boldsymbol{x}_{\theta}$ along the inscribed curve $\left.\operatorname{Vor}\right|_{e}(X) \cap \Sigma$ so that the new candidate triangle satisfies $\rho \leq \bar{\rho}$. These constraints lead to an associated altitude 

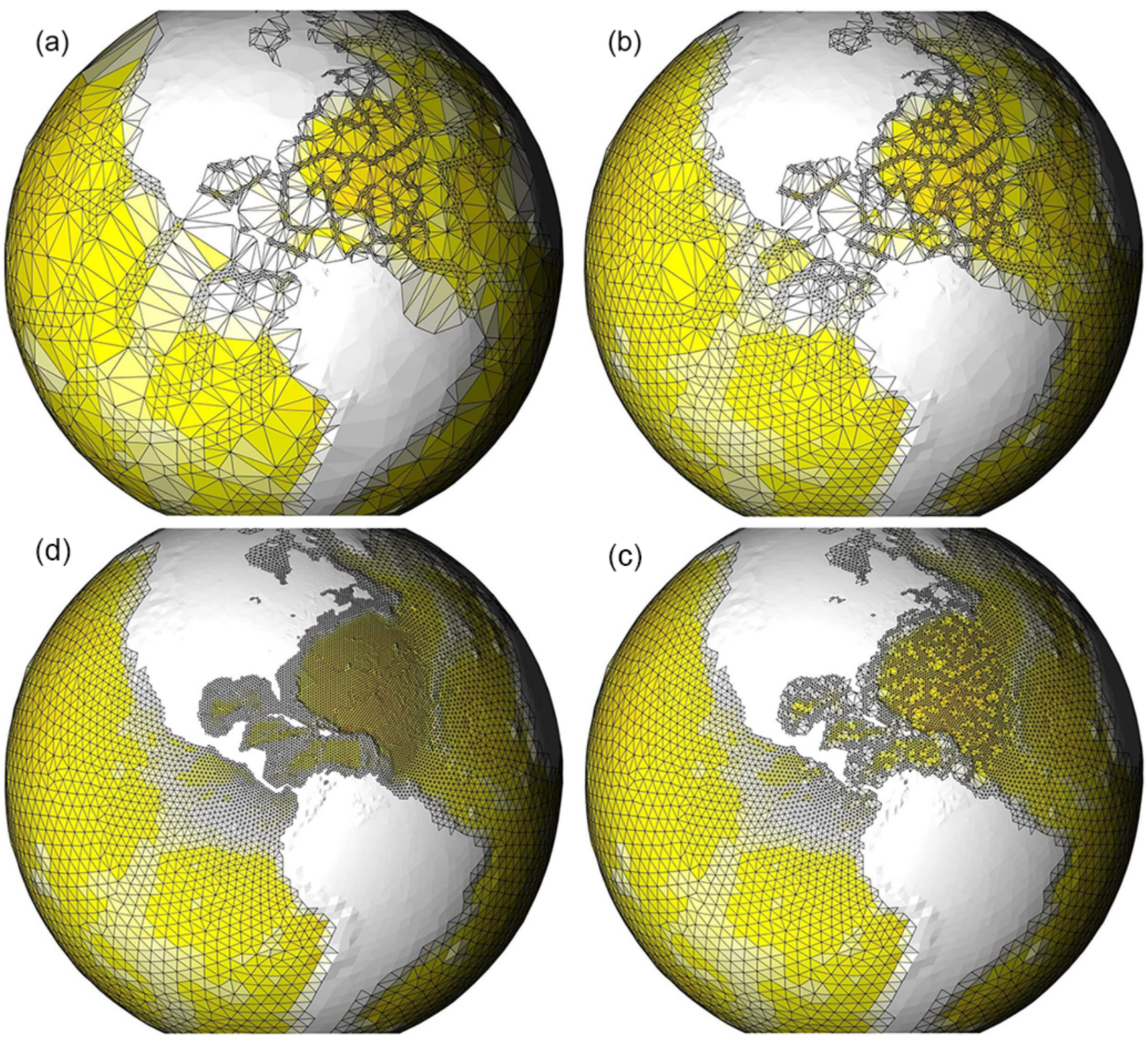

Figure 4. Progress of the Frontal-Delaunay refinement algorithm, clockwise from top left, showing the space-filling refinement trajectory.
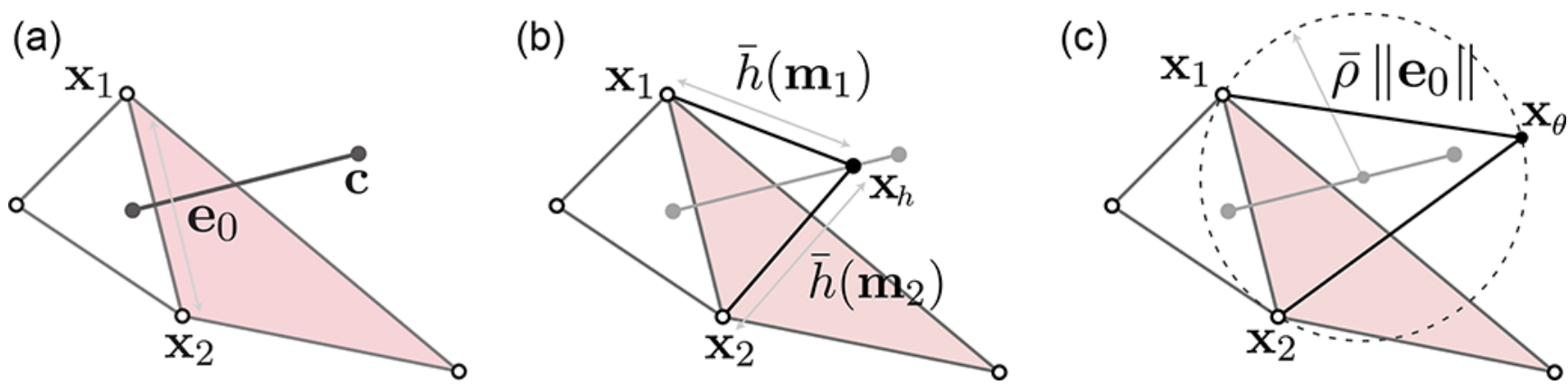

Figure 5. Two-dimensional representation of the off-centre point-placement rules, illustrating (a) a low-quality triangle $f_{i}$ (shaded) to be eliminated, highlighting the frontal segment of the Voronoi diagram associated with the short edge $e_{0} \in f_{i}$, (b) placement of the size-optimal point $\boldsymbol{x}_{h}$ to satisfy local size constraints $\bar{h}\left(\boldsymbol{x}_{f}\right)$, and (c) placement of a shape-optimal point $\boldsymbol{x}_{\theta}$ to satisfy local radius-edge ratios $\bar{\rho}$. 
$a_{\theta}=\frac{\left\|\boldsymbol{e}_{0}\right\|}{\widetilde{\beta}_{\theta}}$,

where

$\widetilde{\beta}_{\theta}=2 \tan \left(\frac{\tilde{\theta}}{2}\right), \quad \tilde{\theta}=\arcsin \left(\frac{1}{2 \bar{\rho}}\right)$.

Given $a_{h}$ and $a_{\theta}$, the position of the points $\boldsymbol{x}_{h}$ and $\boldsymbol{x}_{\theta}$ is calculated by computing the intersection of balls of radius $a_{h}$ and $a_{\theta}$, centred at the midpoint of the frontal edge $e_{0}$ and the frontal curve $\left.\operatorname{Vor}\right|_{e}(X) \cap \Sigma$. This approach ensures that new vertices are positioned by advancing a specified distance along the surface $\Sigma$ in the frontal direction. For non-uniform $\bar{h}(x)$, expressions for the position of the point $\boldsymbol{x}_{h}$ are nonlinear, with the altitude $a_{h}$ depending on an evaluation of the mesh-size function at the edge midpoints $\bar{h}\left(\boldsymbol{m}_{i}\right)$ and visa versa. In practice, since $\bar{h}(\boldsymbol{x})$ is guaranteed to be smooth, a simple iterative predictor-corrector procedure is sufficient to solve these expressions approximately.

Finally, given the set of candidate off-centre vertices $\left\{\boldsymbol{x}_{c}, \boldsymbol{x}_{h}, \boldsymbol{x}_{\theta}\right\}$, the position of the refinement point $\boldsymbol{x}$ for the triangle $f_{i}$ is selected. A worst-first strategy is adopted, choosing the point that satisfies local constraints in a greedy fashion. Specifically, the closest point lying on the adjacent Voronoi segment $\left.\operatorname{Vor}\right|_{e}(X)$ and outside the neighbourhood of the frontal edge $e_{0}$ is selected, with

$\boldsymbol{x}= \begin{cases}\boldsymbol{x}_{j}, & \text { if }\left(d_{j} \leq d_{c} \text { and } d_{j} \geq \frac{1}{2}\left\|\boldsymbol{e}_{0}\right\|\right), \\ \boldsymbol{x}_{c}, & \text { otherwise }\end{cases}$

where $j=\operatorname{argmin}\left(d_{h}, d_{\theta}\right)$, and $d_{j}=\operatorname{dist}\left(\boldsymbol{x}_{e}, \boldsymbol{x}_{j}\right)$ are distances from the midpoint of the frontal edge $e_{0}$ to the sizeand shape-optimal points $\boldsymbol{x}_{h}$ and $\boldsymbol{x}_{\theta}$, respectively. This cascading selection criteria seeks a balance between local optimality and global convergence - smoothly degenerating to a conventional circumcentre-based Delaunay refinement strategy in limiting cases, while using locally optimal points where possible. Specifically, these constraints guarantee that refinement points always lie within a local safe region on the Voronoi complex - being positioned on an adjacent Voronoi segment and bound between the circumcentre of the element itself and the diametric ball of the associated frontal edge. This condition ensures that new points are never positioned too close to an existing vertex, leading to provable guarantees on the performance of the algorithm. See previous work by the author (Engwirda and Ivers, 2016; Engwirda, 2016) for additional detail.

\subsection{Additional remarks}

As a restricted Delaunay refinement approach, a full threedimensional Delaunay tetrahedralisation $\operatorname{Del}(X)$ is incrementally maintained throughout the surface meshing phase.
As per definition 1 , the set of restricted surface triangles $\left.\operatorname{Del}\right|_{\Sigma}(X)$ that lie on the spheroid $\Sigma$, are expressed as a subset of the faces of this tetrahedral complex, such that $\left.\operatorname{Del}\right|_{\Sigma}(X) \subseteq \operatorname{Del}(X)$. In an effort to minimise the expense of maintaining this full-dimensional tessellation, an additional scaffolding vertex $\boldsymbol{x}_{\mathrm{s}}$ is initially inserted at the centre of the spheroid. This has the effect of simplifying the topological structure of $\operatorname{Del}(X)$, with the resulting tetrahedral elements forming a simple wheel-like configuration - emanating radially outward from the scaffolding vertex $\boldsymbol{x}_{\mathrm{s}}$. See Fig. 3 for additional detail.

As shown in Fig. 4, one interesting characteristic of the Frontal-Delaunay algorithm described here relates to the refinement trajectory that is followed when inserting new vertices and triangles. Unlike standard Delaunay refinement or advancing-front-type methods, it can be seen that the algorithm adopts a space-filling curve-type pattern, covering the surface in a fractal-like configuration, before recursively filling in the gaps. Note that no explicit space-filling curve constraint has been implemented here - this behaviour is simply an emergent property of the algorithm itself, and is due to interactions between the greedy priority schedule, the frontal filtering and the off-centre point-placement strategies. In practice, it has been found that this space-filling-type behaviour leads to the construction of very high-quality triangulations, typically exceeding the performance of standard advancing-front or Delaunay refinement-type schemes.

\section{Hill-climbing mesh optimisation}

While the Delaunay grids produced by the refinement algorithm described previously are guaranteed to be of very highquality, producing triangulations with angles bounded between $\theta_{f}=30^{\circ}$ and $\theta_{f}=120^{\circ}$, these tessellations can often be further improved through subsequent mesh-optimisation operations. Recalling that the construction of well-centred grids appropriate for unstructured $\mathrm{C}$-grid schemes require that maximum angles be bounded below $\theta_{f}=90^{\circ}$, the application of such optimisation procedures can in fact be seen as a necessary component of the grid-generation work flow explored here.

In the present work, grid optimisation is realised as a coupled geometrical and topological optimisation task - seeking to reposition vertices and update grid topology to maximise a given element-wise mesh-quality metric $\mathcal{Q}_{f}(\boldsymbol{x})$. A hill-climbing-type optimisation strategy is pursued, in which a locally optimal solution is sought based on an initial grid configuration. In this study, the grid is optimised according to the area-length-quality metric (see definition 3), a robust scalar measure of grid-quality that achieves a score of +1 for perfect elements - decreasing toward zero with increasing levels of element distortion. Optimisation predicates are implemented following a hill-climbing strategy (Freitag and Ollivier-Gooch, 1997; Klingner and Shewchuk, 2008), 
with modifications to the grid accepted only if the local mesh-quality metrics are sufficiently improved. Specifically, a worst-first strategy is adopted here, in which each given operation is required to improve the worst-case quality associated with elements in the local subset acted upon. Such a philosophy ensures that global mesh quality is increased monotonically as optimisation proceeds. Note that such behaviour is designed to maximise the quality of the worst elements in the grid, rather than improving a mean measure. This represents an important distinction when compared to other iterative mesh-optimisation algorithms, such as Centroidal Voronoi Tessellation (CVT)-type schemes (Jacobsen et al., 2013), in which all entities in the grid are adjusted simultaneously until a global convergence criterion is satisfied.

\section{1 "Spring"-based mesh smoothing}

Considering firstly the geometric optimality of the grid, a mesh-smoothing procedure is undertaken - seeking to reposition the nodes of the grid to improve element quality and mesh-spacing conformance. Following the work of Persson and Strang (2004), a spring-based approach is pursued, in which edges in the Delaunay triangulation are treated as elastic rods with a prescribed natural length. Nodes are iteratively repositioned until a local equilibrium configuration is reached. See Algorithm 2 for full details. In the original work of Persson, nodal positions are adjusted via a local timestepping loop, with all nodes updated concurrently under the action of explicit spring forces. In the current study, a noniterative variant is employed, in which each node is repositioned one by one, such that constraints in each local neighbourhood are satisfied directly. Specifically, a given node $\boldsymbol{x}_{i}$ is repositioned as a weighted sum of contributions from incident edges

$\boldsymbol{x}_{i}^{n+1}=\frac{\sum w_{k}\left(\boldsymbol{x}_{i}^{n}+\Delta_{k} \boldsymbol{v}_{k}\right)}{\sum w_{k}}$,

where

$\boldsymbol{v}_{k}=\boldsymbol{x}_{i}^{n}-\boldsymbol{x}_{j}^{n} \quad$ and $\quad \Delta_{k}=\frac{\bar{h}\left(\boldsymbol{x}_{k}^{n}\right)-l_{k}}{l_{k}}$.

Here, $x_{i}^{n}, x_{j}^{n}$ are the current positions of the two nodes associated with the $k$-th edge, $l_{k}$ is the edge length and $\bar{h}\left(\boldsymbol{x}_{k}\right)$ is the value of the mesh-spacing function evaluated, at the edge midpoint. $\Delta_{k}$ is the relative spring extension required to achieve equilibrium in the $k$-th edge. The scalars $w_{k} \in \mathbb{R}^{+}$ are edge weights. Setting $w_{k}=1$ results in an unweighted scheme, consisting of simple linear springs. In this study, the use of non-linear weights, defined by setting $w_{k}=\Delta_{k}^{2}$, was found to offer superior performance.

Noting that application of the spring-based operator (Eq. 11) may move nodes away from the underlying spheroidal surface $\Sigma$, an additional projection operator is introduced to ensure that the grid conforms to the surface geometry exactly. Following the application of each springbased adjustment, nodes are moved back onto the geometry via a closest-point projection.

Consistent with the hill-climbing paradigm described previously, each nodal adjustment is required to be validated before being committed to the updated grid configuration. Specifically, nodal adjustments are accepted only if there is sufficient improvement in the mesh-quality metrics associated with the set of adjacent elements. A sorted comparison of quality metrics before and after nodal repositioning is performed, with nodal adjustments successful if grid quality is improved in a worst-first manner. This lexicographicalquality comparison is consistent with the methodology employed in Klingner and Shewchuk (2008).

\subsection{Gradient-based mesh smoothing}

While the spring-based smoothing operator is effective in adjusting a grid to satisfy mesh-spacing constraints, and tends to improve the quality of the grid on average, it is not guaranteed to improve element quality in all cases. Therefore, an additional steepest ascent strategy is pursued (Freitag and Ollivier-Gooch, 1997), in which nodal positions are adjusted based on the local gradients of incident element-quality functions. See Algorithm 2 for full details. Specifically, a given node $\boldsymbol{x}_{i}$ is repositioned along a local search vector chosen to improve the quality of the worst incident element

$\boldsymbol{x}_{i}^{n+1}=\boldsymbol{x}_{i}^{n}+\Delta_{f} \hat{\boldsymbol{v}}_{f}$,

where

$\boldsymbol{v}_{f}=\frac{\partial}{\partial \boldsymbol{x}}\left(\mathcal{Q}_{f}\left(\boldsymbol{x}_{i}\right)\right), \quad f=\arg \min _{j}\left(\mathcal{Q}_{j}\left(\boldsymbol{x}_{i}\right)\right)$.

Here, the index $j$ is taken as a loop over the Delaunay triangles $\left.f_{j} \in \operatorname{Del}\right|_{\Sigma}(X)$ incident to the node $\boldsymbol{x}_{i}$. The scalar step length $\Delta_{f} \in \mathbb{R}^{+}$is computed via a line search along the gradient ascent vector $\boldsymbol{v}_{f}$ and, in this study, is taken as the first value that leads to an improvement in the worst-case incident-quality metric $\mathcal{Q}_{f}\left(\boldsymbol{x}_{i}\right)$. This length is computed by a simple bisection strategy, iteratively testing $\Delta_{f}^{p}=\left(\frac{1}{2}\right)^{p} \alpha$ until a successful nodal adjustment is found. Here $p$ denotes the local line-search iteration, while the scalar length $\alpha \in \mathbb{R}^{+}$ is determined as a solution to the first-order Taylor expansion

$\mathcal{Q}_{f}\left(\boldsymbol{x}_{i}\right)+\alpha \hat{\boldsymbol{v}}_{f} \cdot \frac{\partial}{\partial \boldsymbol{x}}\left(\mathcal{Q}_{f}\left(\boldsymbol{x}_{i}\right)\right) \leq \widetilde{\mathcal{Q}}_{j}\left(\boldsymbol{x}_{i}\right)$.

The quantity $\widetilde{\mathcal{Q}}_{j}\left(\boldsymbol{x}_{i}\right)$ is the second-lowest grid-quality score associated with the set of triangles $\left.f_{j} \in \operatorname{Del}\right|_{\Sigma}(X)$ incident to the central node $\boldsymbol{x}_{i}$. This selection strategy (Freitag and Ollivier-Gooch, 1997) is designed to compute an initial displacement $\alpha$ that will improve the worst element in the set until its quality is equal that of its next best neighbour. Noting that such an expansion is only first-order accurate, the 


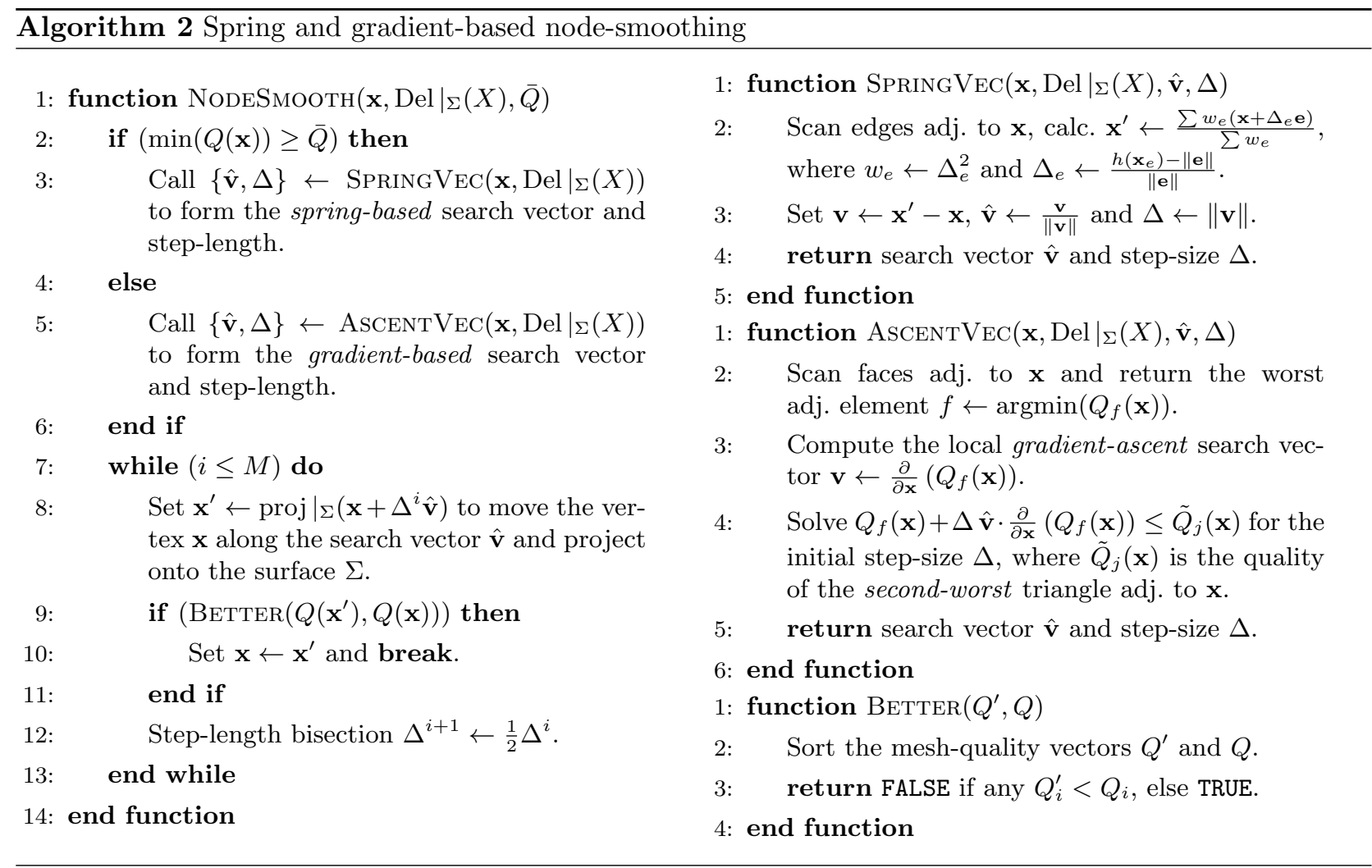

step length is iteratively decreased using the bisection strategy described previously. A limited line search is employed here, testing iterations $p \leq 5$ until a successful step is found. Consistent with the spring-based procedure described previously, a geometry-projection operator is implicitly incorporated within each update (Eq. 13), ensuring that nodes remain constrained to the spheroidal surface.

\subsection{Topological "flips"}

In addition to purely geometrical operations, grid optimisation also requires that adjustments be made to the underlying mesh topology, such that the surface triangulation remains a valid Delaunay structure. While it is possible to simply recompute the full Delaunay tessellation after each adjustment, such an approach would impose significant computational costs, especially when considering that a majority of updates involve small perturbations. In this work, an alternative strategy is pursued, based on local element-wise transformations, known as topological flips.

For any given pair of adjacent surface triangles $\left\{f_{i}, f_{j}\right\} \in$ $\left.\operatorname{Del}\right|_{\Sigma}(X)$, a local re-triangulation can be achieved by flipping the local connectivity about the shared edge $\left\{x_{i}, x_{j}\right\}$, forming a new edge between the opposing vertices $\left\{x_{a}, x_{b}\right\}$. Such an operation results in the deletion of the existing triangles $\left\{f_{i}, f_{j}\right\}$ and the creation of a new pair $\left\{f_{i}^{\prime}, f_{j}^{\prime}\right\}$. This operation is illustrated in Fig. 6a. In the present study, the it- erative application of such edge-flipping operations is used to adjust the topology of the surface triangulation, ensuring that it remains Delaunay throughout the optimisation phase. Specifically, given a general, possibly non-Delaunay, surface triangulation $\left.\operatorname{Tri}\right|_{\Sigma}(X)$, a cascade of edge flips are used to reach a valid restricted Delaunay surface tessellation $\left.\operatorname{Del}\right|_{\Sigma}(X)$. For each adjacent triangle pair $\left\{f_{i}, f_{j}\right\} \in$ $\left.\operatorname{Tri}\right|_{\Sigma}(X)$ an edge flip is undertaken if a local violation of the Delaunay criterion is detected. New elements created by successful edge flips are iteratively re-examined until no further modifications are necessary. This approach follows the standard flip-based algorithms described in, for instance, Lawson (1977); Cheng et al. (2013).

Given a triangle $\left.f_{i} \in \operatorname{Tri}\right|_{\Sigma}(X)$, the local Delaunay criterion is violated if there exists a node $x_{q} \notin f_{i}$ interior to the associated circumscribing ball. In this work, such violations are detected by considering the restricted circumballs $B\left(\boldsymbol{c}_{i}, r_{i}\right)$ associated with each triangle $\left.f_{i} \in \operatorname{Tri}\right|_{\Sigma}(X)$, where the centre of the balls $\boldsymbol{c}_{i}$ are a projection of the planar element circumcentres onto the spheroidal surface $\Sigma$. Such constructions are designed to account for the curvature of the underlying surface. Given an adjacent triangle pair $\left.\left\{f_{i}, f_{j}\right\} \in \operatorname{Tri}\right|_{\Sigma}(X)$ an edge flip is undertaken if either opposing vertex $x_{a}, x_{b}$ lies within the circumball associated with the adjacent triangle. To prevent issues associated with exact floating-point comparisons, a small relative tolerance 
is incorporated. Specifically, nodes are required to penetrate the opposing circumball by a distance $\epsilon$ before an edge flip is undertaken, with $\epsilon=\frac{1}{2}\left(r_{i}+r_{j}\right) \bar{\epsilon}$ and $\bar{\epsilon}=1 \times 10^{-10}$ in the current double-precision implementation.

\subsection{Edge contraction}

In some cases, grid-quality and mesh-spacing conformance can be improved through the application of edge-contraction operations, whereby nodes are removed from the grid by collapsing certain edges. Given an edge $\left.e_{k} \in \operatorname{Tri}\right|_{\Sigma}(X)$, a retriangulation of the local cavity $\left.\mathcal{C}_{i} \subseteq \operatorname{Tri}\right|_{\Sigma}(X)$, formed by the set of triangles incident to the nodes $\left\{x_{i}, x_{j}\right\} \in e_{k}$, can be achieved by merging the nodes $x_{i}$ and $x_{j}$ at some local mean position. In addition to collapsing the edge $e_{k}$, edge contraction also removes the two surface triangles $\left\{f_{i}, f_{j}\right\} \in$ $\left.\operatorname{Tri}\right|_{\Sigma}(X)$ adjacent to $e_{k}$, resulting in a re-triangulation of the local cavity $\left.\mathcal{C}_{i}^{\prime} \subseteq \operatorname{Tri}\right|_{\Sigma}(X)$. See Fig. 6 b for illustration. In the present work, nodes are merged to a mean position $\boldsymbol{x}_{n}$ - taken as the average of adjacent element circumcentres, such that $\boldsymbol{x}_{n}=\frac{1}{\left|\mathcal{C}_{i}\right|} \sum c_{j}$. Here, the $c_{j}$ 's are the centres of the circumballs associated with the triangles $f_{j} \in \mathcal{C}_{i}$. As per previous discussions, the mean position $\boldsymbol{x}_{n}$ is projected onto the spheroidal surface $\Sigma$. While such an approach is slightly more computationally intensive than strategies based on collapsing to edge midpoints, this weighted circumcentrebased scheme was found to be substantially more effective in practice. Consistent with the hill-climbing philosophy pursued throughout this study, edge contraction operations are only utilised if there is sufficient improvement in local meshquality metrics. Specifically, edge contraction is undertaken if the quality of the new cavity $\mathcal{C}_{i}^{\prime}$ exceeds that of the initial configuration $\mathcal{C}_{i}$ in a worst-first fashion.

\subsection{Edge refinement}

Fulfilling the opposite role to edge contraction, edgerefinement operations seek to improve the grid through the addition of new nodes and elements. In the present study, a simplified refinement operation is utilised, in which a given edge $\left.e_{k} \in \operatorname{Tri}\right|_{\Sigma}(X)$ is refined by placing a new node $x_{n}$ at the centre of the restricted circumball $B\left(\boldsymbol{c}_{i}, r_{i}\right)$ associated with the lower-quality adjacent triangle $\left.f_{i} \in \operatorname{Tri}\right|_{\Sigma}(X)$. Insertion of the new node $x_{n}$ induces a re-triangulation of the local cavity $\left.\mathcal{C}_{i} \in \operatorname{Tri}\right|_{\Sigma}(X)$ - constructed by expanding about $x_{n}$ in a local greedy fashion. Starting from the initial cavity $\mathcal{C}_{i}=\left\{f_{i}, f_{j}\right\}$, where $\left.\left\{f_{i}, f_{j}\right\} \in \operatorname{Tri}\right|_{\Sigma}(X)$ are the triangles adjacent to the edge $e_{k}$, additional elements are added in a breadth-first manner, with a new, unvisited neighbour $f_{k}$ added to the cavity $\mathcal{C}_{i}$ if doing so will improve the worst-case element-quality metric. The final cavity $\mathcal{C}_{i}$ is therefore a locally optimal configuration. In practice, this iterative deepening of $\mathcal{C}_{i}$ typically convergences in one or two iterations. See Fig. $6 \mathrm{c}$ for illustration. As per the edge-contraction and nodesmoothing operations described previously, edge refinement is implemented according to a hill-climbing-type philosophy, with operations successful only if there is sufficient improvement in local grid quality. Consistent with previous discussions, a lexicographical comparison of the grid-quality metrics associated with elements in the initial and final states $\mathcal{C}_{i}$ and $\mathcal{C}_{i}^{\prime}$ is used to determine success.

\subsection{Optimisation schedule}

The full grid-optimisation procedure is realised as a combination of the various geometrical and topological operations described previously, organised into a particular iterative optimisation schedule. See Algorithm 3 for full details. Each outer iteration consists of a fixed set of operations: four node-smoothing passes, a single pass of edge refinement/contraction operations, and, finally, iterative edge flipping to restore the Delaunay criterion. In this study, 16 outer iterations are employed. Each node-smoothing pass is a composite operation, with the spring-based technique used to adjust nodes adjacent to high-quality elements, and the gradient-ascent method used otherwise. Specifically, springbased smoothing is used to adjust nodes adjacent to elements with a minimum-quality score of $\overline{\mathcal{Q}}_{f} \geq 0.9375$. Such thresholding ensures that the expensive gradient-ascent-type iteration is reserved for the worst elements in the grid. The optimisation schedule employed here is not based on any rigorous theoretical derivation, but is simply a set of heuristic choices that have proven to be effective in practice. The application of multiple node-smoothing passes within an outer iteration containing subsequent topological, contraction and refinement-type operations is consistent with the methodologies employed in, for instance, Freitag and Ollivier-Gooch (1997); Klingner and Shewchuk (2008).

\section{Results and discussions}

The performance of the Frontal-Delaunay refinement and hill-climbing optimisation algorithms presented in Sects. 3 and 4 was investigated experimentally, with the methods used to mesh a series of benchmark problems. The algorithm was implemented in $\mathrm{C}++$ and compiled as a 64 bit executable. The full algorithm has been implemented as a specialised variant of the general-purpose JIGSAW meshing package, denoted JIGSAW-GEO, and is currently available online (Engwirda, 2017) or by request from the author. All tests were completed on a Linux platform using a single core of an Intel i7 processor. Visualisation and post-processing was completed using MATLAB.

\subsection{Preliminaries}

A set of three benchmark problems were considered. The UNIFORM-SPHERE test case is a fixed resolution meshing problem on the sphere, suitable for uniformly resolved atmospheric and/or oceanic simulations. The REGIONAL- 

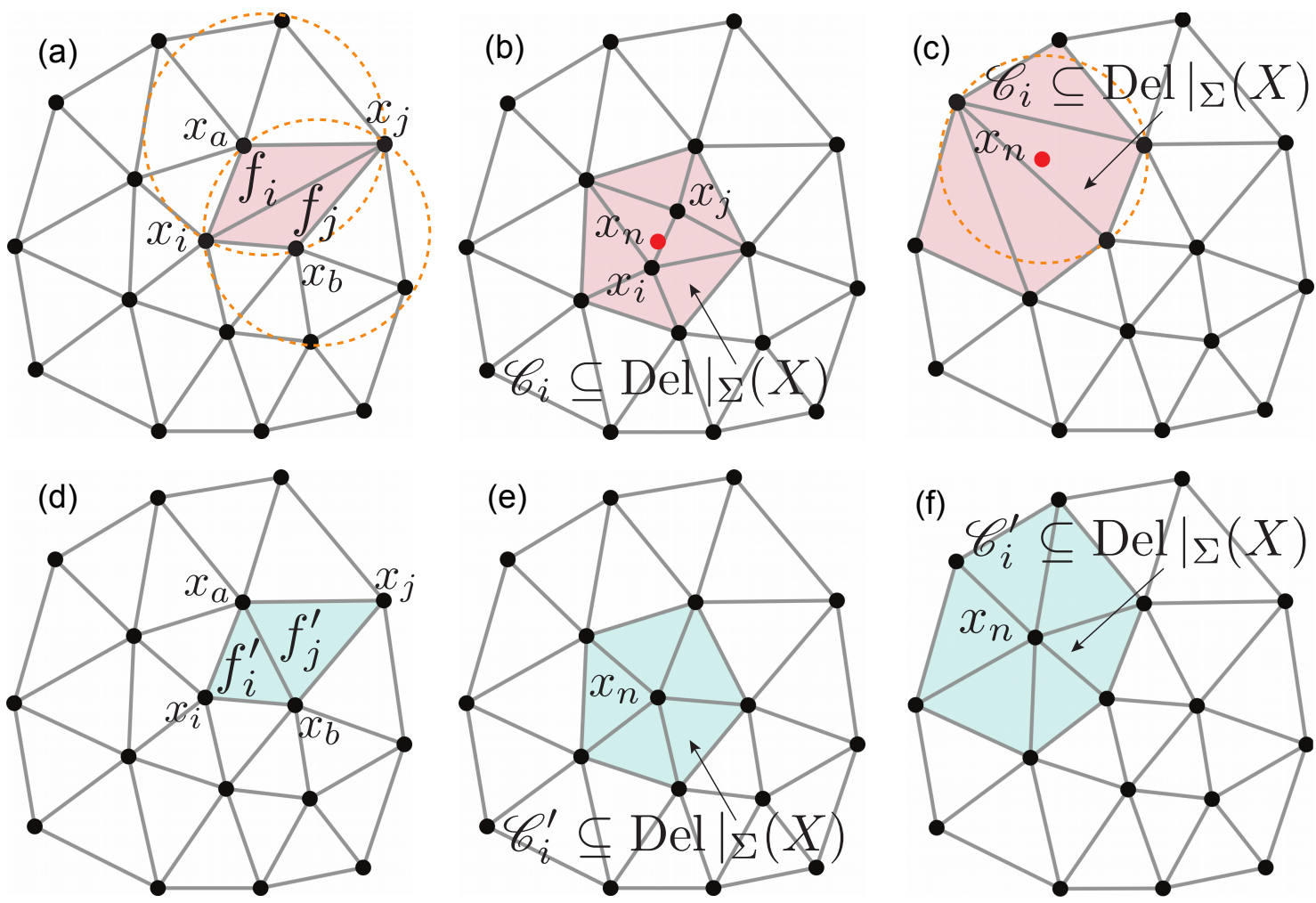

Figure 6. Topological operations for grid optimsiation, showing (a, d) an edge flip, (b, e) an edge contraction, and (c, f) an edge-refinement operation. Grid configurations before and after each flip are shown in the upper and lower panels, respectively.

ATLANTIC test case explores simple, regional refinement for ocean modelling, embedding a high-resolution, eddypermitting representation of the North Atlantic ocean basin in a coarse global grid. The SOUTHERN-OCEAN test case is designed to test the multi-resolution capabilities of the algorithm, defining a complex non-uniform grid-spacing function for the Southern Ocean and Antarctic regions. The meshspacing constraints for this problem were designed to incorporate a combination of topographic and regional refinement. The Voronoi-Delaunay grids for these test cases are shown in Figs. 8, 10, 13, and 15, with the associated gridquality statistics presented in Figs. 9, 11, and 14. The underlying grid-spacing definitions used for the REGIONALATLANTIC and SOUTHERN-OCEAN problems are shown in Fig. 7.

In all test cases, limiting radius-edge ratios were specified, with $\bar{\rho}_{f}=1.05$. This constraint ensures that the minimum angles in the Delaunay triangulations are bounded, with $\theta_{\min } \geq 28.4^{\circ}$. For all test problems, detailed statistics on element quality are presented, including histograms of element area-length ratios $a_{f}$, element angles $\theta_{f}$, and relative-edge lengths $h_{\mathrm{r}}$. These quality metrics are described in detail in Sect. 3.4. Histograms highlight the minimum, maximum, and mean values of the relevant distributions as appropriate.

\subsection{Uniform global grid}

The performance of the JIGSAW-GEO algorithm was first assessed using the UNIFORM-SPHERE test case, seeking to build a uniformly resolved global grid for general circulation modelling. Spatially uniform mesh-size constraints were enforced, setting $\bar{h}(\boldsymbol{x})=150 \mathrm{~km}$ over the full sphere. The resulting grid is shown in Fig. 8 and contains 83072 Delaunay triangles and 41538 Voronoi cells. Grid-quality metrics are presented in Fig. 9, showing distributions before and after the application of the grid-optimisation procedure.

Visual inspection of these grids show that very high levels of geometric quality are achieved, with both the Delaunay triangulation and Voronoi tessellation consisting of highly regular elements, free of obvious distortion and/or areas of overor under-refinement. Focusing on the element shape-quality statistics, it is noted that very high levels of mesh regularity are achieved, with the vast majority of element area-length scores tightly clustered about $a_{f}=1$. Similarly, the distribution of element angles shows strong convergence around $\theta_{f}=60^{\circ}$, revealing most triangles to be near equilateral. Finally, analysis of the relative-length distribution shows that edge lengths follow the imposed mesh-spacing constraints closely, with very tight clustering about $h_{\mathrm{r}}=1$.

The effect of the grid-optimisation procedure can be assessed by comparing the mesh-quality statistics presented in 

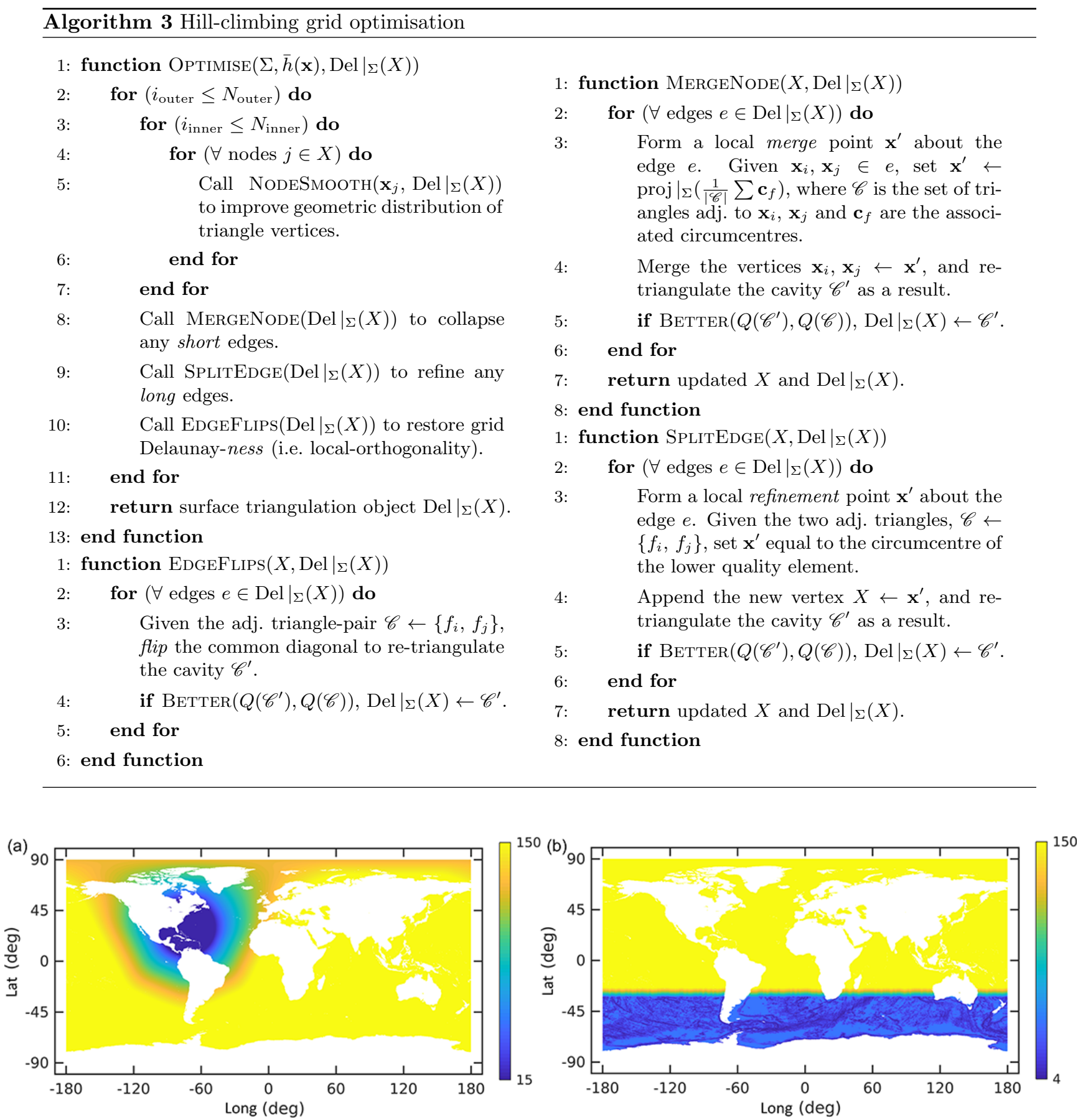

Figure 7. Mesh-spacing functions $\bar{h}(\boldsymbol{x})$ for the regionally refined North Atlantic and topographically refined Southern Ocean grids. Mesh spacing is shown in kilometres.

Fig. 9. The application of mesh optimisation is seen to be most pronounced at the tails of the distributions, showing that, as expected, the hill-climbing-type procedure is effective at improving the worst elements in the grid. Specifically, the minimum area-length metric is improved from $a_{f}=0.67$ to $a_{f}=0.94$, and the distribution of element-wise angles is narrowed from $31^{\circ} \leq \theta_{f} \leq 112^{\circ}$ to $44^{\circ} \leq \theta_{f} \leq 78^{\circ}$. A slight broadening of the mean parts of the distributions is also evident, showing that in some cases, higher-quality elements are slightly compromised to facilitate improvements to their lower-quality neighbours. This behaviour is consistent with the worst-first philosophy employed in this study. 

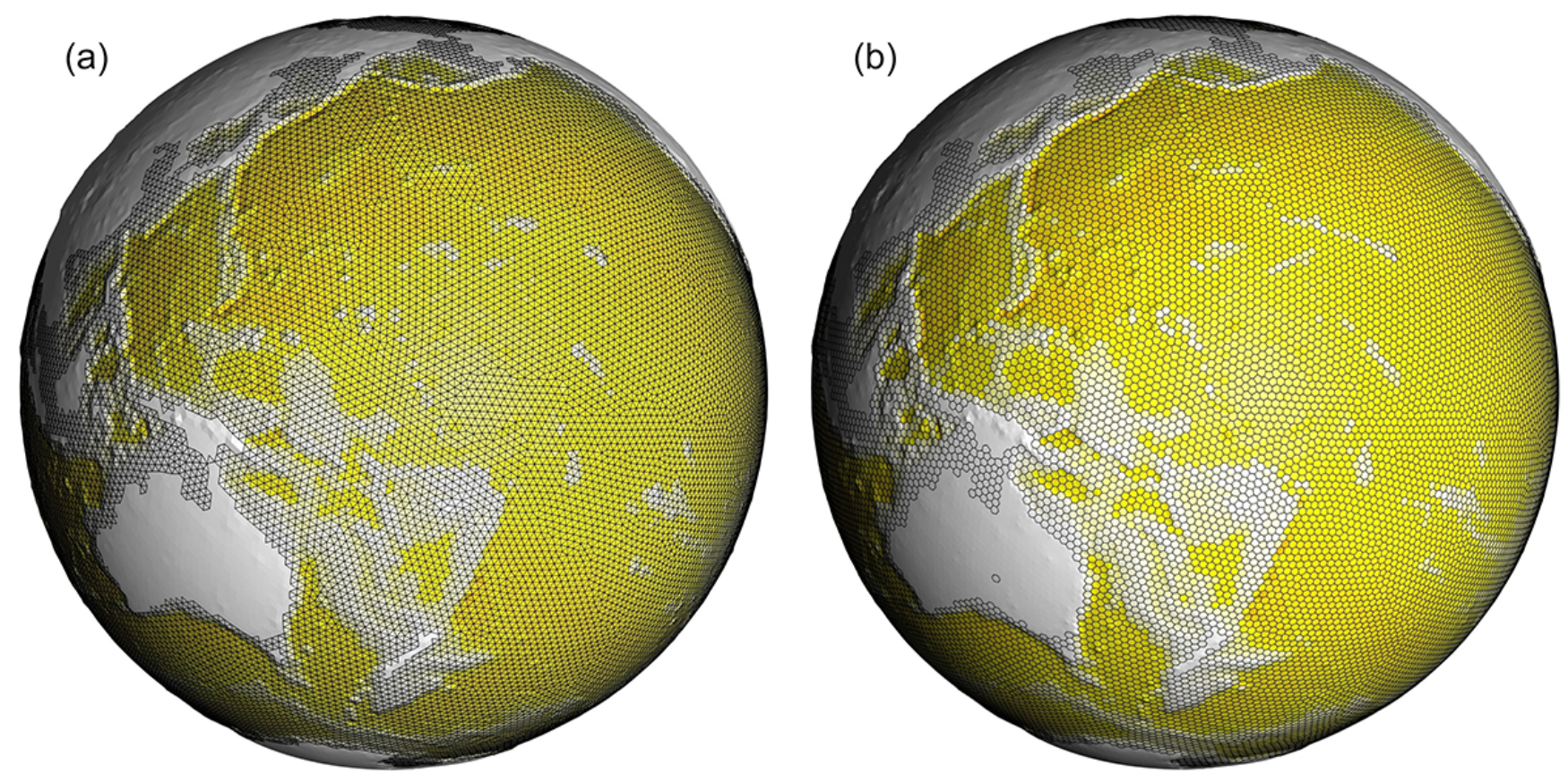

Figure 8. A uniform resolution global grid, showing (a) the underlying spheroidal Delaunay triangulation, and (b) the associated staggered Voronoi dual; $150 \mathrm{~km}$ grid spacing was specified globally. Topography is drawn using an exaggerated scale, with elevation from the reference geoid amplified by a factor of 10 .

(a)

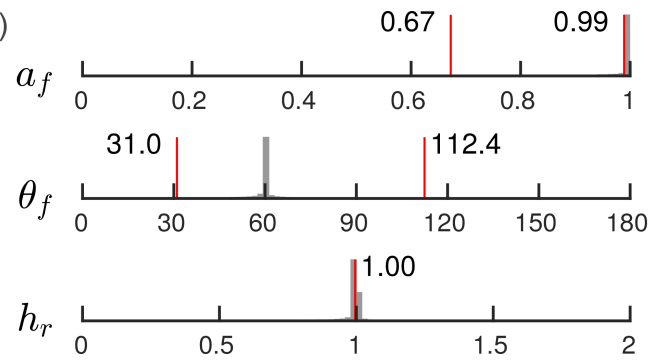

(b)

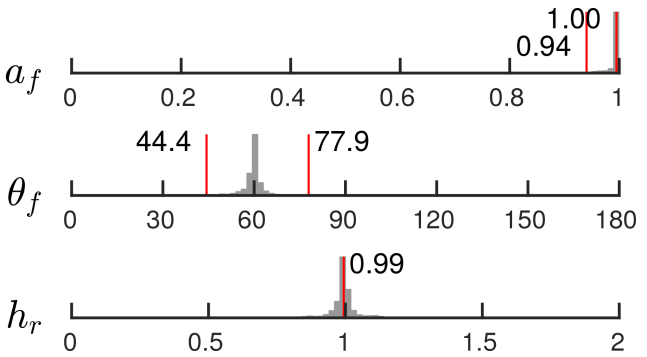

Figure 9. Mesh-quality metrics for the uniform resolution grid, before (a) and after (b) the application of mesh optimisation. Histograms of area-length ratio $a_{f}$, enclosed-angle $\theta_{f}$ and relative-length ratio $h_{\mathrm{r}}$ are illustrated, with min., max., and mean values annotated.

Beyond improvements to grid-quality statistics, the impact of mesh optimisation can be further understood by considering the well centredness of the resulting staggered VoronoiDelaunay grid. Well-centred triangulations are those for which all element circumcentres are located within their parent triangles, ensuring that the associated Voronoi cells are nicely staggered with respect to the underlying triangulation as a result. Such a constraint is equivalent to requiring that all Delaunay triangles are acute, with $\theta_{f} \leq 90^{\circ}$. Further details regarding these constraints are presented in Sect. 2.

Well-centred grids are highly desirable from a numerical perspective, allowing for, for instance, the unstructured Cgrid scheme employed in the MPAS framework to achieve optimal rates of convergence. Specifically, when a grid is well centred, it is guaranteed that associated edges in the staggered Voronoi-Delaunay cells intersect, ensuring that evaluation of the cell transport and circulation terms can be accurately computed using compact numerical stencils. In the case of perfectly centred grids, such intersections occur at edge midpoints - allowing for a numerical scheme based on local linear interpolants to achieve second-order accuracy.

The construction of well-centred grids is known to be a difficult problem, and the development of algorithms for their generation is an ongoing area of research (VanderZee et al., 2008; Vanderzee et al., 2010). For the uniform-resolution case studied here, it is clear that the JIGSAW-GEO algorithm is successful in generating such a grid, with all angles bounded below $77.9^{\circ}$.

\subsection{Regionally refined North Atlantic grid}

The multi-resolution capabilities of the JIGSAW-GEO algorithm were investigated in the REGIONAL-ATLANTIC test 


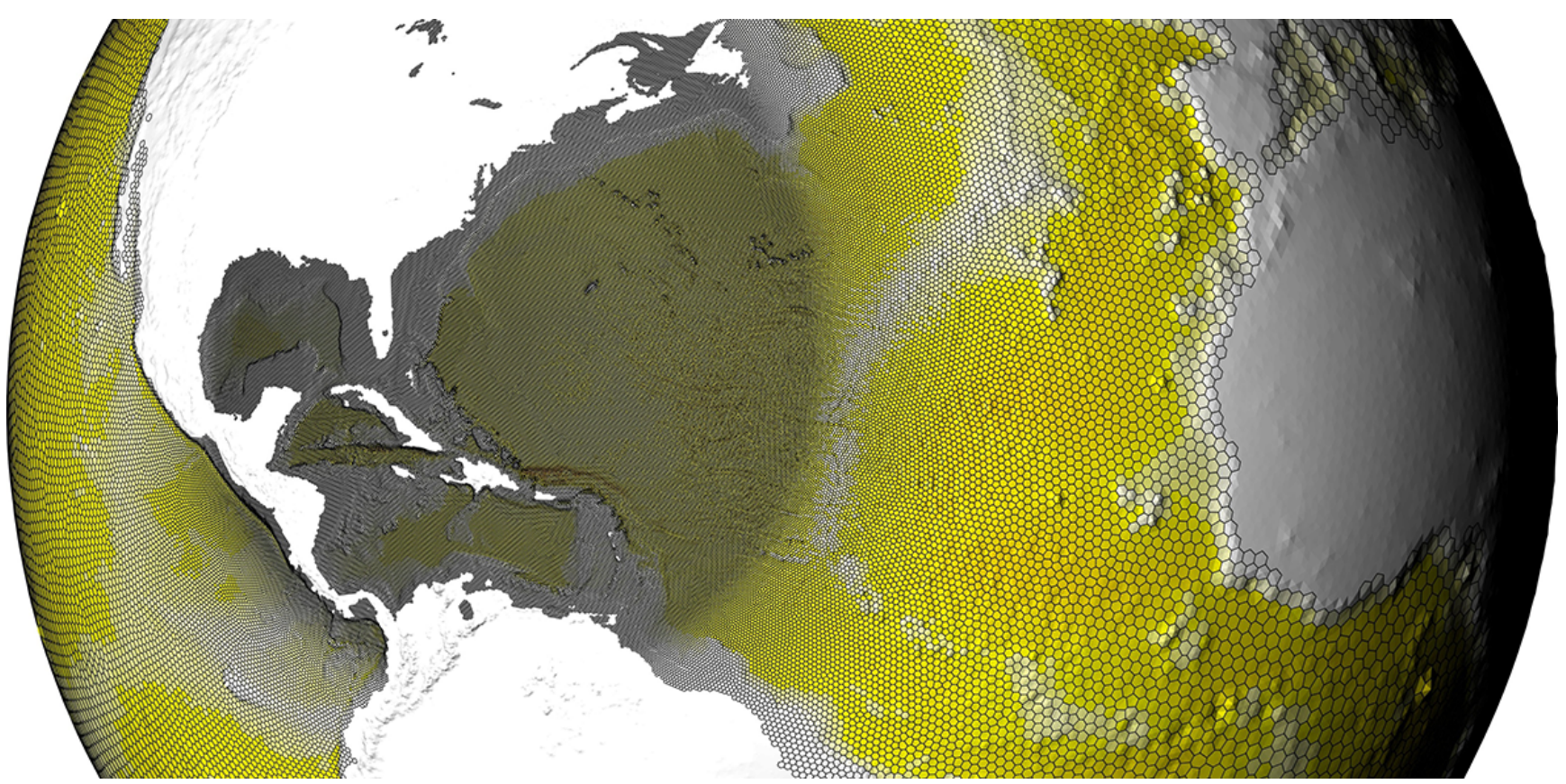

Figure 10. A regionally refined Voronoi-type grid of the North Atlantic region. Global coarse grid resolution is $150 \mathrm{~km}$, with a $15 \mathrm{~km}$ eddypermitting grid-spacing specified over the Atlantic ocean basin. Topography is drawn using an exaggerated scale, with elevation from the reference geoid amplified by a factor of 10 .

(a)

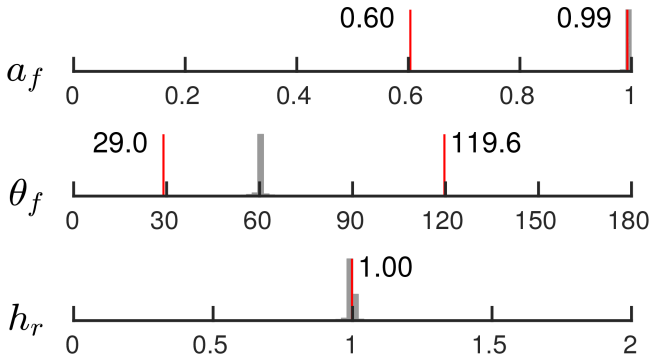

(b)

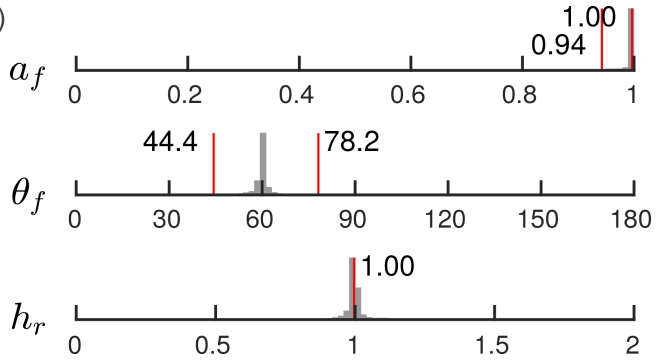

Figure 11. Mesh-quality metrics for the regionally refined grid, before (a) and after (b) the application of mesh optimisation. Histograms of area-length ratio $a_{f}$, enclosed-angle $\theta_{f}$ and relative-length ratio $h_{\mathrm{r}}$ are illustrated, with min., max., and mean values annotated.

case, seeking to build a regionally resolved grid for highresolution modelling of the North Atlantic Ocean basin. Nonuniform mesh-size constraints were enforced, setting $\bar{h}(\boldsymbol{x})=$ $150 \mathrm{~km}$ globally, with $15 \mathrm{~km}$ eddy-permitting mesh spacing specified over the North Atlantic region. The resulting grid is shown in Figs. 10 and 12 and contains 358064 Delaunay triangles and 179081 Voronoi cells. Grid-quality metrics are presented in Fig. 11, showing distributions before and after the application of grid optimisation.

Consistent with the results presented previously, a very high-quality Voronoi-Delaunay grid was generated for the REGIONAL-ATLANTIC problem, with each grid-quality metric tightly clustered about its optimal value, such that $a_{f} \rightarrow 1, \theta_{f} \rightarrow 60^{\circ}$, and $h_{\mathrm{r}} \rightarrow 1$. As per the uniform resolution case, the application of mesh optimisation led to significant improvements in worst-case grid quality, with the minimum area-length metric improved from $a_{f}=0.60$ to $a_{f}=0.94$, and the distribution of element angles narrowed from $29^{\circ} \leq \theta_{f} \leq 120^{\circ}$ to $44^{\circ} \leq \theta_{f} \leq 78^{\circ}$. The resulting optimised grid is also clearly well centred, with all angles in the Delaunay triangulation less than $78.2^{\circ}$. Overall, grid quality can be seen to achieve essentially the same levels of optimality as the uniform resolution test case, showing that the JIGSAW-GEO algorithm can be used to generate very highquality spatially adaptive grids without obvious degradation in mesh quality.

\subsection{Multi-resolution Southern Ocean grid}

The JIGSAW-GEO algorithm was then used to mesh the challenging SOUTHERN-OCEAN test case, allowing its performance for large-scale problems involving rapidly vary- 


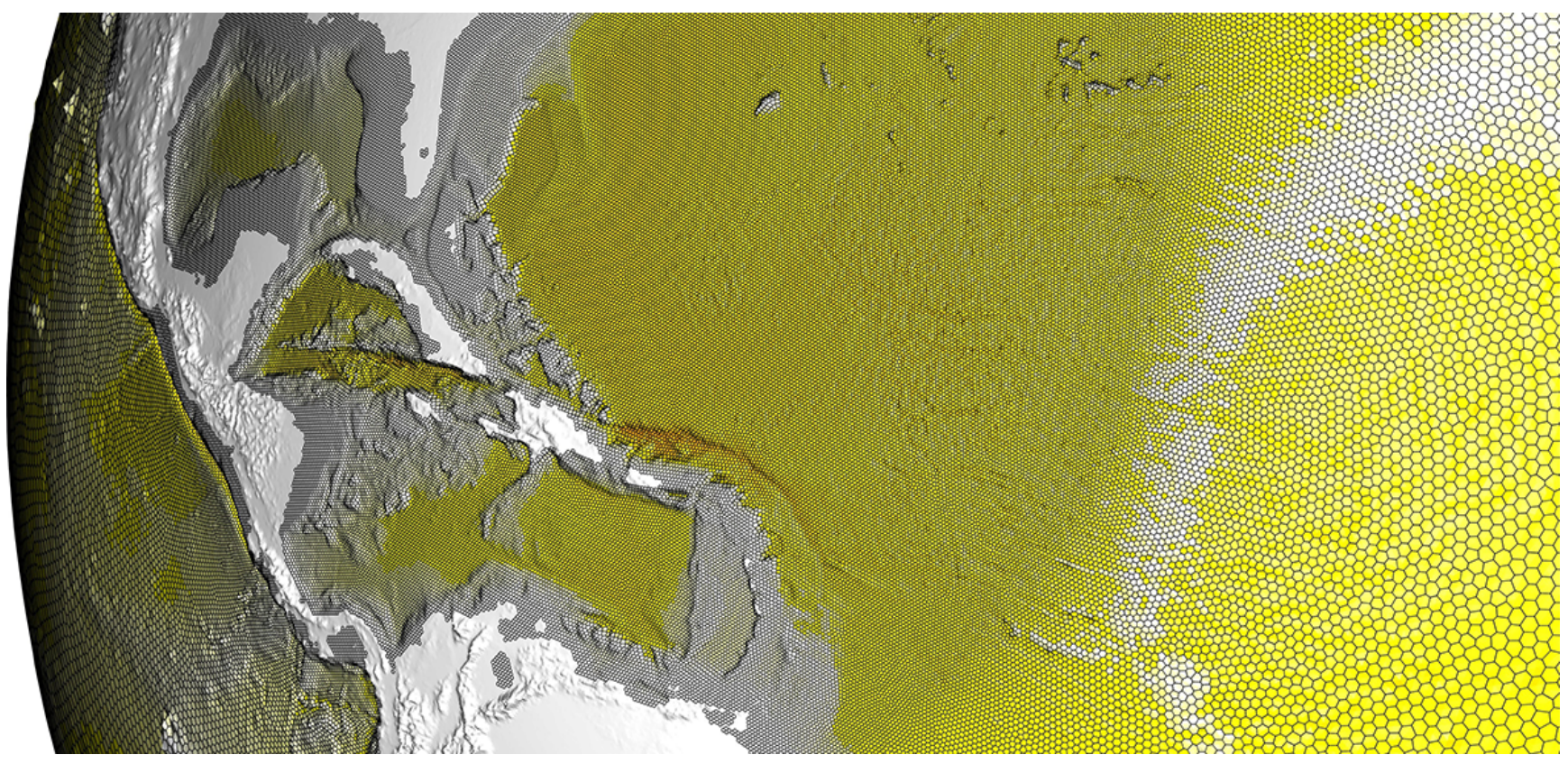

Figure 12. Additional detail of the regionally refined North Atlantic grid shown in Fig. 10.

ing mesh-spacing constraints to be analysed in detail. This test case seeks to build a multi-resolution grid for regionally refined ocean studies, with a particular focus on resolution of the Antarctic Circumpolar Current, and adjacent Antarctic processes. Composite mesh-spacing constraints were enforced, consisting of a coarse global background resolution of $150 \mathrm{~km}$, with an eddy-permitting $15 \mathrm{~km}$ grid spacing specified south of $32.5^{\circ} \mathrm{S}$. An additional topographic adaptation was also utilised within the southern annulus, with grid resolution increased in regions of large bathymetric gradient. A minimum grid spacing of $4 \mathrm{~km}$ was specified. Topographic gradients were computed using the high-resolution ETOPO1 Global Relief dataset (Amante and Eakins, 2009). The resulting grid is shown in Fig. 13, with additional detail shown in Fig. 15. The grid contains 3119849 Delaunay triangles and 1559927 Voronoi cells. Associated grid-quality metrics are presented in Fig. 14, showing distributions before and after the application of the grid-optimisation procedure.

Consistent with previous results, visual inspection of Figs. 13 and 15 confirm that the JIGSAW-GEO algorithm is capable of generating very high-quality multi-resolution grids, containing a majority of near-perfect Delaunay triangles and Voronoi cells. Additionally, it can be seen that grid resolution varies smoothly, even in regions of rapidly fluctuating mesh-spacing constraints (see Fig. 15 for detail). Analysis of the grid-quality metrics shown in Fig. 14 show that very high levels of mesh regularity are achieved, with element area-length scores tightly clustered about $a_{f}=$ 1 and element angles showing strong convergence around $\theta_{f}=60^{\circ}$. Interestingly, despite the complexity of the imposed mesh-spacing function, the relative-length distribution still shows relatively tight conformance, with a sharp clustering about $h_{\mathrm{r}}=1$. Overall, mean grid quality is slightly reduced compared to the uniform resolution case, illustrated by a slight broadening of the grid-quality distributions. Note that such behaviour is expected in the multi-resolution case, with imperfect triangle geometries required to satisfy nonuniform mesh-spacing constraints.

The effect of the grid-optimisation procedure can be assessed by comparing the mesh-quality statistics presented in Fig. 14. As per the uniform resolution case, mesh optimisation appears to be most aggressive at the tails of the distributions, acting to improve the worst elements in the grid. The minimum area-length metric is improved from $a_{f}=0.59$ to $a_{f}=0.90$, and the distribution of element angles is narrowed from $28^{\circ} \leq \theta_{f} \leq 121^{\circ}$ to $40^{\circ} \leq \theta_{f} \leq 80^{\circ}$. Consistent with previous results, a moderate broadening of the mean components of the distributions can be observed, especially in the enclosed-angle and relative-length metrics. As per previous discussions, this behaviour shows that some high-quality elements are slightly compromised to improve their lowerquality neighbours.

The optimised grid is also clearly well centred, with all angles in the Delaunay triangulation bounded below $80^{\circ}$. This result shows only marginal degradation compared to the simpler examples presented previously - demonstrating the effectiveness of the optimisation strategies described here. These results show that very high-quality, well-centred grids can be generated for complex multi-resolution cases, even when the grid-spacing function incorporates strong local fluctuation. Nonetheless, the construction of well-centred grids remains a challenging task, and it is noted that there 


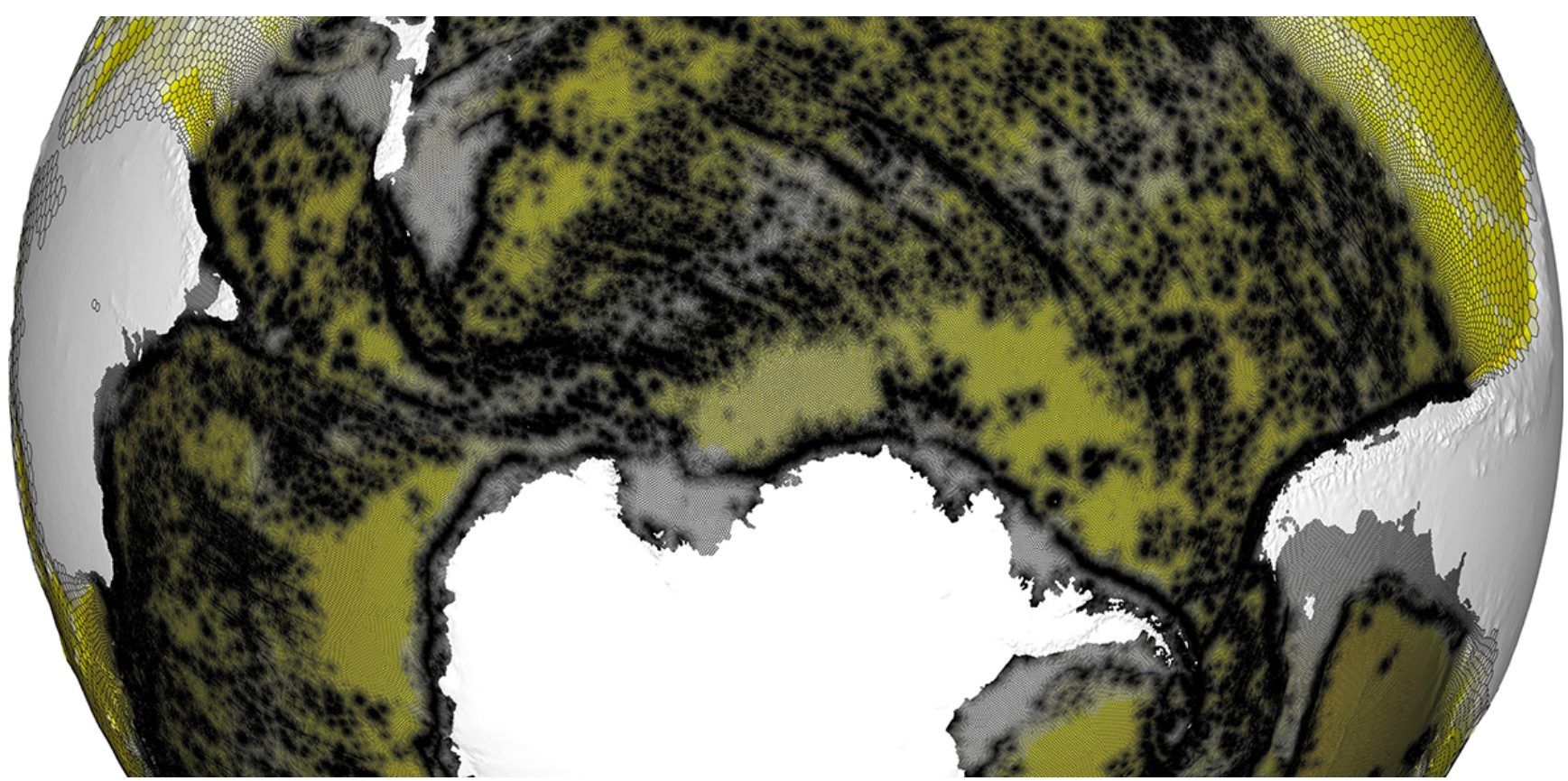

Figure 13. A multi-scale grid for the Southern Ocean. Coarse resolution is $150 \mathrm{~km}$, with $15 \mathrm{~km}$ eddy-permitting grid spacing specified south of $32.5^{\circ} \mathrm{S}$. Local topographic adaptation is also utilised, with resolution increased in areas of large bathymetric gradient. Minimum grid spacing is $4 \mathrm{~km}$. Topography is drawn using an exaggerated scale, with elevation from the reference geoid amplified by a factor of 10 .

(a)

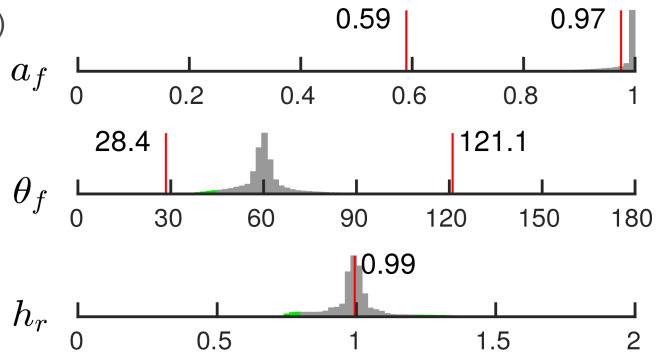

(b)

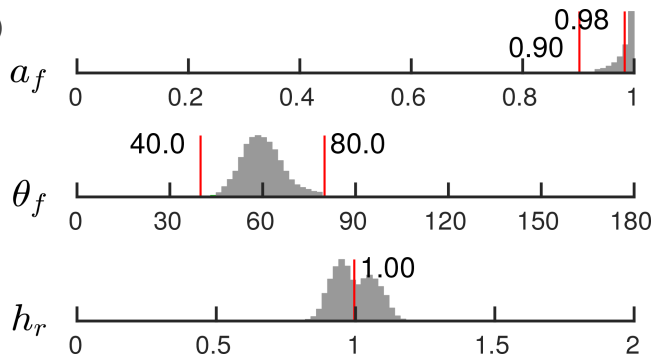

Figure 14. Mesh-quality metrics associated with the multi-resolution grid, before (a) and after (b) the application of mesh optimisation. Histograms of area-length ratio $a_{f}$, enclosed-angle $\theta_{f}$ and relative-length ratio $h_{\mathrm{r}}$ are illustrated, with min., max., and mean values annotated.

may exist test cases that defeat the current strategy. Therefore, the pursuit of alternative optimisation techniques, designed to target grid well-centredness directly, is an interesting avenue for future research.

\subsection{Computational performance}

In addition to the generation of very high-quality grids, the new JIGSAW-GEO algorithm also imposes a relatively moderate computational burden, producing large-scale, multi-resolution grids in a matter of minutes using standard desktop-based computing infrastructure. Specifically, grid-generation for the UNIFORM-SPHERE, REGIONALATLANTIC, and SOUTHERN-OCEAN test cases required approximately $12 \mathrm{~s}, 1 \frac{1}{2} \mathrm{~min}$, and $10 \mathrm{~min}$ of computation time, respectively, running on a single core of an Intel i7 processor. In all cases, grid optimisation was found to be approxi- mately 4 times as expensive as the initial Frontal-Delaunay refinement. Compared to the existing iterative MPI-SCVT algorithm (Jacobsen et al., 2013), commonly used to generate grids for the MPAS framework, these results represent a significant increase in productivity, with the MPI-SCVT algorithm often requiring days, or even weeks of distributed computing time.

Additionally, practical experience with the MPI-SCVT algorithm has shown that it cannot always be relied upon to generate an appropriate grid, irrespective of the amount of computational time allowed for convergence to be reached. While always generating locally orthogonal and centroidal tessellations with very high mean grid qualities, the MPISCVT algorithm does not provide bounds on the worst-case metrics. In practice, multi-resolution grids generated using the MPI-SCVT algorithm are often observed to contain a mi- 


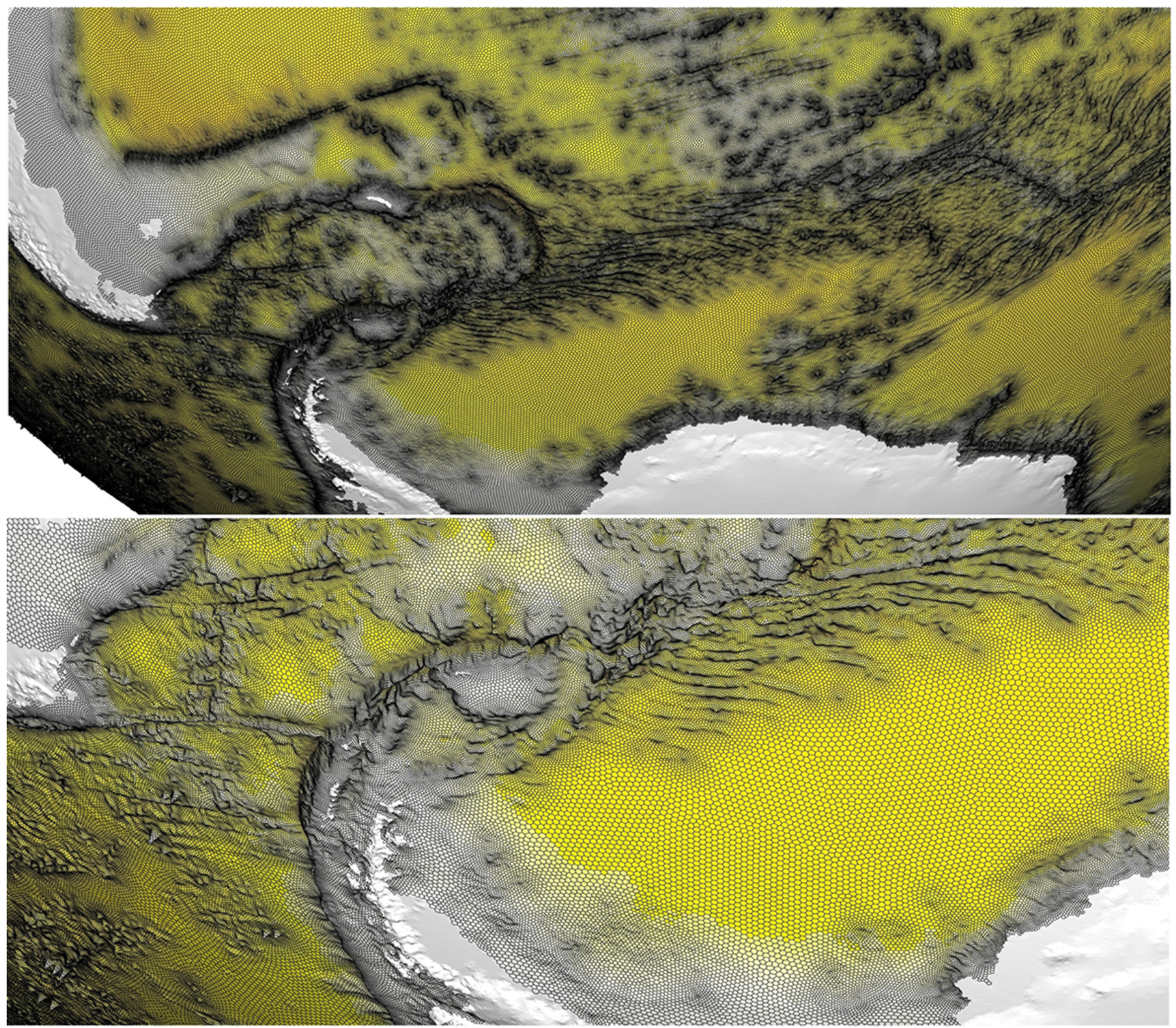

Figure 15. Additional detail of the multi-resolution Southern Ocean grid shown in Fig. 13.

nority of obtuse triangles that violate the well-centred constraint. As per the discussions presented in Sect. 2, such grids are inappropriate for use in an unstructured C-grid model such as MPAS. Currently, grid generation for such models often requires a degree of user-driven trial and error as a result, making grid generation a somewhat arduous task for model users. Initial experiments conducted using the JIGSAW-GEO algorithm have shown it to be a useful alternative, reliably generating valid well-centred multi-resolution grids for a wide range of user-defined constraints and configuration settings. Initial evaluation of JIGSAW-GEO for use with the MPAS framework is currently underway.

\section{Conclusions and future work}

A new algorithm for the generation of multi-resolution staggered unstructured grids for large-scale general circulation modelling on the sphere has been described. Using a combination of Frontal-Delaunay refinement and hill-climbingtype optimisation techniques, it has been shown that very high-quality locally orthogonal, centroidal, and well-centred spheroidal grids appropriate for unstructured C-grid-type general circulation models can be generated. The performance of this new approach has been verified using a number of multi-scale global benchmarks, including difficult problems incorporating highly non-uniform mesh-spacing constraints. 
This new algorithm is available as part of the JIGSAW meshing package, providing a simple and easy-to-use tool for the oceanic and atmospheric modelling communities. A number of benchmark problems have been analysed, examining the performance of the new approach. The FrontalDelaunay refinement algorithm has been shown to generate guaranteed-quality spheroidal Delaunay triangulations satisfying worst-case bounds on element angles and exhibiting smooth grading characteristics. This algorithm has been shown to produce very high-quality multi-resolution triangulations, with a majority of elements exhibiting strong conformance to element-shape and grid-spacing constraints.

The use of a coupled geometrical and topological hillclimbing optimisation procedure was shown to further improve grid quality, especially for the lowest quality elements in each mesh. It was demonstrated that these optimisation techniques allow for grid quality to be improved to the extent that fully well-centred mesh configurations can be achieved, with angles in the Delaunay triangulation bounded below $90^{\circ}$. For the three global test cases presented here, angles were bounded above $\theta_{f} \geq 40^{\circ}$ and below $\theta_{f} \leq 80^{\circ}$.

The construction of meshes appropriate for a range of contemporary unstructured C-grid-type general circulation mod- els was also discussed in detail, with a focus on the generation of multi-resolution grids for the MPAS framework. The availability of this new algorithm is expected to significantly reduce the grid-generation burden for MPAS model users.

Future work will focus on a generalisation of the algorithm and improvements to its efficiency, including (i) support for coastal constraints, (ii) improvements to computational performance through parallelism, and (iii) further enhancements to the mesh-optimisation procedures, with a focus on improving the well centredness of the resulting staggered grids. The investigation of solution-adaptive multi-scale representations, in which grid resolution is adapted to spatial variability in model state (Sein et al., 2016), is also an obvious direction for future investigation.

Code availability. The JIGSAW-GEO grid generator used in this study is available as a Zenodo archive: https://doi.org/10.5281/zenodo.556602 (Engwirda, 2017). The JIGSAW-GEO framework is under active development and the latest version can be accessed here: https://github.com/dengwirda/jigsaw-geo-matlab. 


\section{Appendix A: Spheroidal predicates}

Computation of the restricted Delaunay surface tessellation $\left.\operatorname{Del}\right|_{\Sigma}(X)$ requires the evaluation of a single predicate. Given a spheroidal surface $\Sigma$, the task is to compute intersections between edges in the Voronoi tessellation $\operatorname{Vor}(X)$ and the surface $\Sigma$.

\section{Restricted surface triangles}

Restricted surface triangles $\left.f_{i} \in \operatorname{Del}\right|_{\Sigma}(X)$ are defined as those associated with an intersecting Voronoi edge $v_{e} \in$ $\operatorname{Vor}(X)$, where $v_{e} \cap \Sigma \neq \varnothing$. These triangles provide a good piecewise linear approximation to the surface $\Sigma$. For a given triangle $f_{i}$, the associated Voronoi edge $v_{e}$ is defined as the line-segment joining the two circumcentres $\boldsymbol{c}_{i}$ and $\boldsymbol{c}_{j}$ associated with the pair of tetrahedrons that share the face $f_{i}$. The task then is to find intersections between the line-segments $v_{e}$ and the surface $\Sigma$. Let $\boldsymbol{p}$ be a point on a given Voronoi edge-segment $v_{e}$

$\boldsymbol{p}=\overline{\boldsymbol{c}}+t \Delta, \quad-1 \leq t \leq+1$, where

$\bar{c}=\frac{1}{2}\left(c_{i}+c_{j}\right) \quad$ and $\quad \Delta=\frac{1}{2}\left(c_{j}-c_{i}\right)$.

Substituting Eq. (A1) into the equation of the spheroidal surface $\Sigma$ leads to a quadratic expression for the parameter $t$

$$
\begin{aligned}
& \sum_{i=1}^{3}\left(\frac{\overline{\boldsymbol{c}}_{i}+t \Delta_{i}}{r_{i}}\right)^{2}=1, \\
& \sum_{i=1}^{3} \frac{\overline{\boldsymbol{c}}_{i}^{2}+2 t \overline{\boldsymbol{c}}_{i} \Delta_{i}+t^{2} \Delta_{i}^{2}}{r_{i}^{2}}=1, \\
& \sum_{i=1}^{3}\left(\frac{\Delta_{i}^{2}}{r_{i}^{2}}\right) t^{2}+\left(\frac{2 \overline{\boldsymbol{c}}_{i} \Delta_{i}}{r_{i}^{2}}\right) t+\left(\frac{\overline{\boldsymbol{c}}_{i}^{2}}{r_{i}^{2}}-1\right)=0 .
\end{aligned}
$$

Any real solutions $-1 \leq t_{\Sigma} \leq+1$ correspond to non-trivial intersections $v_{e} \cap \Sigma \neq \varnothing$. The corresponding point of intersection $\boldsymbol{p}_{\Sigma}$ can be found by substituting $t_{\Sigma}$ into Eq. (A1). 
Competing interests. The authors declare that they have no conflict of interest.

Acknowledgements. This work was carried out at the NASA Goddard Institute for Space Studies, the Massachusetts Institute of Technology, and the University of Sydney with the support of a NASA-MIT cooperative agreement and an Australian Postgraduate Award. The author wishes to thank Todd Ringler, Luke Van Roekel, Mark Petersen, Matthew Hoffman, and Phillip Wolfram for their assistance on grid generation for the MPAS-O environment. John Marshall provided feedback on an earlier version of the manuscript. The author also wishes to thank the anonymous reviewers for their helpful comments and feedback.

Edited by: S. Marras

Reviewed by: two anonymous referees

\section{References}

Adcroft, A., Campin, J.-M., Hill, C., and Marshall, J.: Implementation of an atmosphere-ocean general circulation model on the expanded spherical cube, Mon. Weather Rev., 132, 2845-2863, 2004.

Amante, C. and Eakins, B. W.: ETOPO1 1 Arc-Minute Global Relief Model: Procedures, Data Sources and Analysis, NOAA technical memorandum, NESDIS NGDC-24, https://doi.org/10.7289/V5C8276M , 2009.

Arakawa, A. and Lamb, V. R.: Computational design of the basic dynamical processes of the UCLA general circulation model, Methods in computational physics, 17, 173-265, 1977.

Bernard, P.-E., Chevaugeon, N., Legat, V., Deleersnijder, E., and Remacle, J.-F.: High-order h-adaptive discontinuous Galerkin methods for ocean modelling, Ocean Dynam., 57, 109-121, 2007.

Bleck, R.: An oceanic general circulation model framed in hybrid isopycnic-Cartesian coordinates, Ocean Model., 4, 55-88, 2002.

Bochev, P. B. and Hyman, J. M.: Principles of mimetic discretizations of differential operators, in: Compatible spatial discretizations, 89-119, Springer New York, USA, 2006.

Boissonnat, J. D. and Oudot, S.: Provably Good Surface Sampling and Approximation, in: ACM International Conference Proceeding Series, 43, 9-18, 2003.

Boissonnat, J. D. and Oudot, S.: Provably Good Sampling and Meshing of Surfaces, Graph. Models, 67, 405-451, 2005.

Chen, C., Liu, H., and Beardsley, R. C.: An unstructured grid, finitevolume, three-dimensional, primitive equations ocean model: application to coastal ocean and estuaries, J. Atmos. Ocean. Tech., 20, 159-186, 2003.

Chen, C., Huang, H., Beardsley, R. C., Liu, H., Xu, Q., and Cowles, G.: A finite volume numerical approach for coastal ocean circulation studies: Comparisons with finite difference models, J. Geophys. Res.-Oceans, 112, 2007.

Cheng, S. W., Dey, T. K., Ramos, E. A., and Ray, T.: Sampling and Meshing a Surface with Guaranteed Topology and Geometry, SIAM J. Comput., 37, 1199-1227, 2007.
Cheng, S. W., Dey, T. K., and Ramos, E. A.: Delaunay Refinement for Piecewise Smooth Complexes, Discrete Comput. Geom., 43, 121-166, https://doi.org/10.1007/s00454-008-9109-3, 2010.

Cheng, S. W., Dey, T. K., and Shewchuk, J. R.: Delauay Mesh Generation, Taylor \& Francis, New York, USA, 2013.

Comblen, R., Legrand, S., Deleersnijder, E., and Legat, V.: A finite element method for solving the shallow water equations on the sphere, Ocean Model., 28, 12-23, 2009.

Conroy, C. J., Kubatko, E. J., and West, D. W.: ADMESH: An advanced, automatic unstructured mesh generator for shallow water models, Ocean Dynam., 62, 1503-1517, 2012.

Du, Q., Faber, V., and Gunzburger, M.: Centroidal Voronoi tessellations: applications and algorithms, SIAM Rev., 41, 637-676, 1999.

Edelsbrunner, H. and Shah, N. R.: Triangulating Topological Spaces, Int. J. Comput. Geom. App., 7, 365-378, 1997.

Engwirda, D.: Conforming restricted Delaunay mesh generation for piecewise smooth complexes, Procedia Eng., 163, 84-96, 2016.

Engwirda, D.: JIGSAW-GEO: Grid-generation for general circulation modelling on the sphere (Version 1.0.1), https://doi.org/10.5281/zenodo.556602, 2017.

Engwirda, D. and Ivers, D.: Off-centre Steiner points for Delaunay-refinement on curved surfaces, 23rd International Meshing Roundtable Special Issue: Advances in Mesh Generation, Comput. Aided Design, 72, 157-171, https://doi.org/10.1016/j.cad.2015.10.007, 2016.

Erten, H. and Üngör, A.: Quality Triangulations with Locally Optimal Steiner Points, SIAM J. Sci. Comp., 31, 2103-2130, 2009.

Ford, R., Pain, C., Piggott, M., Goddard, A., De Oliveira, C., and Umpleby, A.: A nonhydrostatic finite-element model for threedimensional stratified oceanic flows. Part I: model formulation, Mon. Weather Rev., 132, 2816-2831, 2004a.

Ford, R., Pain, C., Piggott, M., Goddard, A., de Oliveira, C., and Umpleby, A.: A nonhydrostatic finite-element model for threedimensional stratified oceanic flows. Part II: model validation, Mon. Weather Rev., 132, 2832-2844, 2004b.

Freitag, L. A. and Ollivier-Gooch, C.: Tetrahedral mesh improvement using swapping and smoothing, Int. J. Numer. Meth. Eng., 40, 3979-4002, 1997.

Frey, J. L. and George, P. L.: Mesh Generation: Applications to Finite Elements, Hermés, Paris, France, 2000.

Fringer, O., Gerritsen, M., and Street, R.: An unstructured-grid, finite-volume, nonhydrostatic, parallel coastal ocean simulator, Ocean Model., 14, 139-173, 2006.

Geuzaine, C. and Remacle, J.-F.: Gmsh: A 3-D finite element mesh generator with built-in pre-and post-processing facilities, Int. J. Numer. Meth. Eng., 79, 1309-1331, 2009.

Heikes, R. and Randall, D. A.: Numerical integration of the shallow-water equations on a twisted icosahedral grid. Part I: Basic design and results of tests, Mon. Weather Rev., 123, 18621880, 1995.

Holleman, R., Fringer, O., and Stacey, M.: Numerical diffusion for flow-aligned unstructured grids with application to estuarine modeling, Int. J. Numer. Meth. Fl., 72, 1117-1145, 2013.

Jacobsen, D. W., Gunzburger, M., Ringler, T., Burkardt, J., and Peterson, J.: Parallel algorithms for planar and spherical Delaunay construction with an application to centroidal Voronoi tessellations, Geosci. Model Dev., 6, 1353-1365, https://doi.org/10.5194/gmd-6-1353-2013, 2013. 
Jamin, C., Alliez, P., Yvinec, M., and Boissonnat, J.D.: CGALmesh: A Generic Framework for Delaunay Mesh Generation, ACM T. Math. Software, 41, 1-24, https://doi.org/10.1145/2699463, 2015.

Klingner, B. M. and Shewchuk, J. R.: Aggressive Tetrahedral Mesh Improvement, in: Proceedings of the 16th international meshing roundtable, edited by: Brewer, M. L. and Marcum D., Springer, Berlin, Heidelberg, Germany, 3-23, 2008.

Lai, Z., Chen, C., Cowles, G. W., and Beardsley, R. C.: A nonhydrostatic version of FVCOM: 1. Validation experiments, J. Geophys. Res.-Oceans, 115, C11010, https://doi.org/10.1029/2009JC005525, 2010.

Lambrechts, J., Comblen, R., Legat, V., Geuzaine, C., and Remacle, J.-F.: Multiscale mesh generation on the sphere, Ocean Dynam., 58, 461-473, 2008.

Lawson, C. L.: Software for $C^{1}$ Surface Interpolation, in: Mathematical Software III, edited by: Rice, J. R., 161-194, Academic Press, New York, USA, 1977.

Lipnikov, K., Manzini, G., and Shashkov, M.: Mimetic finite difference method, J. Comput. Phys., 257, 1163-1227, 2014.

Löhner, R.: Regridding surface triangulations, J. Comput. Phys., 126, 1-10, 1996.

Madec, G.: NEMO ocean engine (version 3.3), Tech. Rep. 27, Note du Pole de moélisation, Institut Pierre-Simon Laplace (IPSL), Paris, France, 2011.

Marshall, J., Adcroft, A., Hill, C., Perelman, L., and Heisey, C.: A finite-volume, incompressible Navier-Stokes model for studies of the ocean on parallel computers, J. Geophys. Res., 102, 57535766, 1997.

Murray, R. J.: Explicit generation of orthogonal grids for ocean models, J. Comput. Phys., 126, 251-273, 1996.

Pain, C., Piggott, M., Goddard, A., Fang, F., Gorman, G., Marshall, D., Eaton, M., Power, P., and De Oliveira, C.: Three-dimensional unstructured mesh ocean modelling, Ocean Model., 10, 5-33, 2005.

Persson, P.-O.: Mesh Size Functions for Implicit Geometries and PDE-based Gradient Limiting, Eng. Comput., 22, 95-109, https://doi.org/10.1007/s00366-006-0014-1, 2006.

Persson, P.-O. and Strang, G.: A simple mesh generator in MATLAB, SIAM Rev., 46, 329-345, 2004.

Piggott, M., Gorman, G., Pain, C., Allison, P., Candy, A., Martin, B., and Wells, M.: A new computational framework for multiscale ocean modelling based on adapting unstructured meshes, Int. J. Numer. Meth. Fl., 56, 1003-1015, 2008.

Putman, W. M. and Lin, S.-J.: Finite-volume transport on various cubed-sphere grids, J. Comput. Phys., 227, 55-78, 2007.

Randall, D. A., Ringler, T. D., Heikes, R. P., Jones, P., and Baumgardner, J.: Climate modeling with spherical geodesic grids, Comput. Sci. Eng., 4, 32-41, 2002.
Rebay, S.: Efficient Unstructured Mesh Generation by Means of Delaunay Triangulation and Bowyer-Watson Algorithm, J. Comput. Phys., 106, 125-138, https://doi.org/10.1006/jcph.1993.1097, 1993.

Ringler, T., Ju, L., and Gunzburger, M.: A multiresolution method for climate system modeling: application of spherical centroidal Voronoi tessellations, Ocean Dynam., 58, 475-498, 2008.

Ringler, T., Thuburn, J., Klemp, J. B., and Skamarock, W. C.: A unified approach to energy conservation and potential vorticity dynamics for arbitrarily-structured C-grids, J. Comput. Phys., 229, 3065-3090, 2010.

Ringler, T., Petersen, M., Higdon, R. L., Jacobsen, D., Jones, P. W., and Maltrud, M.: A multi-resolution approach to global ocean modeling, Ocean Model., 69, 211-232, 2013.

Rypl, D. and Krysl, P.: Triangulation of 3D surfaces, Eng. Comput., 13, 87-98, 1997.

Schreiner, J., Scheidegger, C. E., Fleishman, S., and Silva, C. T.: Direct (Re)Meshing For Efficient Surface Processing, Comput. Graph. Forum, 25, 527-536, https://doi.org/10.1111/j.14678659.2006.00972.x, 2006.

Sein, D. V., Danilov, S., Biastoch, A., Durgadoo, J. V., Sidorenko, D., Harig, S., and Wang, Q.: Designing variable ocean model resolution based on the observed ocean variability, J. Adv. Model. Earth Syst., 8, 904-916, https://doi.org/10.1002/2016MS000650, 2016.

Skamarock, W. C., Klemp, J. B., Duda, M. G., Fowler, L. D., Park, S.-H., and Ringler, T. D.: A multiscale nonhydrostatic atmospheric model using centroidal Voronoi tesselations and C-grid staggering, Mon. Weather Rev., 140, 3090-3105, 2012.

VanderZee, E., Hirani, A. N., Guoy, D., and Ramos, E.: Wellcentered Planar Triangulation - An Iterative Approach, in: Proceedings of the 16th International Meshing Roundtable, edited by: Brewer, M. L. and Marcum, D., Springer, Berlin, Heidelberg, 121-138, https://doi.org/10.1007/978-3-540-75103-8_7, 2008.

VanderZee, E., Hirani, A. N., Guoy, D., and Ramos, E. A.: Wellcentered triangulation, SIAM J. Sci. Comput., 31, 4497-4523, https://doi.org/10.1137/090748214, 2010.

Vitousek, S. and Fringer, O. B.: A nonhydrostatic, isopycnalcoordinate ocean model for internal waves, Ocean Model., 83, 118-144, 2014.

Wang, Q., Danilov, S., Sidorenko, D., Timmermann, R., Wekerle, C., Wang, X., Jung, T., and Schröter, J.: The Finite Element Sea Ice-Ocean Model (FESOM) v.1.4: formulation of an ocean general circulation model, Geosci. Model Dev., 7, 663693, https://doi.org/10.5194/gmd-7-663-2014, 2014. 